\title{
Transitive Graphs With Fewer Than Twenty Vertices
}

\author{
By Brendan D. McKay
}

\begin{abstract}
A graph is called transitive if its automorphism group acts transitively on the vertex set. We list the 1031 transitive graphs with fewer than 20 vertices, together with many of their properties.
\end{abstract}

1. Introduction. A transitive graph is one whose automorphism group acts transitively on its vertices. In this report we present a catalogue of all transitive graphs with fewer than 20 vertices. The only other catalogue of transitive graphs appears to be that of Yap [5], who considered transitive graphs with 13 or fewer vertices except those of degree 5. Unfortunately, Yap missed three graphs with 12 vertices. Our method of construction was completely different from that of Yap and involved extensive machine computation. However, a description of the constructive method would be too lengthy to include here. It will be presented in a future paper.

The number of transitive graphs found for each order and degree appears in Table 1. The same information, restricted to connected transitive graphs, appears in Table 2. Note that in both tables, the total for each order includes those with degree not in the table. Also, note that a transitive graph with $n$ vertices and degree $d$ is connected if $d \geqslant(n-1) / 2$.

2. Terminology. Basic graph terminology not defined here can be found in Behzad and Chartrand [1]. Suppose $X$ is a graph with vertex set $V(X)=\{1,2, \ldots, n\}$ and edge set $E(X)$. We denote by $\operatorname{Aut}(X)$ the automorphism group of $X$ and by Aut $_{1}(X)$ the stabilizer in $\operatorname{Aut}(X)$ of vertex 1 . We call $X$ transitive if the action of Aut $(X)$ on $V(X)$ is transitive.

Two partitions of $V(X)$ will be defined. Firstly, $\partial(X)$ is the partition of $V(X)$ such that vertices $v$ and $w$ are in the same cell if and only if $\partial(1, v)=\partial(1, w)$, where $\partial(x, y)$ is the distance in $X$ between $x$ and $y$. By convention, $\partial(x, y)=\infty$ if $x$ and $y$ are in different components of $X$. Secondly, $\alpha(X)$ is the partition of $V(X)$ whose cells are the orbits of $\operatorname{Aut}_{1}(X)$. We will say that $X$ is distance regular if, for any cells $C_{1}, C_{2} \in \partial(X)$, not necessarily distinct, and for any $v, w \in C_{1}$, vertex $v$ is adjacent to the same number of vertices in $C_{2}$ as is vertex $w$. We will say that $X$ is distance transitive if $\partial(X)=\alpha(X)$. For connected transitive graphs, these definitions correspond to those of Biggs [2]. It is easy to show that a distance transitive graph is also distance regular, but the converse need not be true.

Received May 24, 1978.

AMS (MOS) subject classifications (1970). Primary 05-04, 05C99; Secondary 05C25. 


\begin{tabular}{|c|c|c|c|c|c|c|c|c|c|c|}
\hline & & & & & gree & & & & & \\
\hline order & 0 & 1 & 2 & 3 & 4 & 5 & $\epsilon$ & 7 & 8 & total \\
\hline 1 & 1 & & & & & & & & & 1 \\
\hline 2 & 1 & 1 & & & & & & & & 2 \\
\hline 3 & 1 & 0 & 1 & & & & & & & 2 \\
\hline 4 & 1 & 1 & 1 & 1 & & & & & & 4 \\
\hline 5 & 1 & 0 & 1 & 0 & 1 & & & & & 3 \\
\hline 6 & 1 & 1 & 2 & 2 & 1 & 1 & & & & 8 \\
\hline 7 & 1 & 0 & 1 & 0 & 1 & 0 & 1 & & & 4 \\
\hline 8 & 1 & 1 & 2 & 3 & 3 & 2 & 1 & $i$ & & 14 \\
\hline 9 & 1 & 0 & 2 & 0 & 3 & 0 & 2 & 0 & 1 & 9 \\
\hline 10 & 1 & 1 & 2 & 3 & 4 & 4 & 3 & 2 & 1 & 22 \\
\hline 11 & 1 & 0 & 1 & 0 & 2 & 0 & 2 & 0 & 1 & 8 \\
\hline 12 & 1 & 1 & 4 & 7 & 11 & 13 & 13 & 11 & 7 & 74 \\
\hline 13 & 1 & 0 & 1 & 0 & 3 & 0 & 4 & 0 & 3 & 14 \\
\hline 14 & 1 & $\mathrm{i}$ & 2 & 3 & $t$ & $\epsilon$ & $\theta$ & 9 & 6 & 56 \\
\hline 15 & 1 & 0 & 3 & 0 & 3 & 0 & 12 & 0 & 12 & 48 \\
\hline 16 & 1 & 1 & 3 & 7 & 16 & 27 & 40 & 48 & 48 & 286 \\
\hline 17 & 1 & 0 & 1 & 0 & 4 & 0 & 7 & 0 & 10 & 36 \\
\hline 18 & 1 & 1 & 4 & 7 & 16 & 24 & 38 & 45 & 54 & 380 \\
\hline 19 & 1 & 0 & 1 & 0 & 4 & 0 & 10 & 0 & 14 & 60 \\
\hline
\end{tabular}

TABLE 1. Number of transitive graphs

Suppose that $X$ has diameter $\delta$, not necessarily finite. Then $X$ will be called antipodal if, for distinct vertices $u, v, w$, we have $\partial(u, v)=\partial(u, w)=\delta$ implies $\partial(v, w)$ $=\delta$. A new graph $D=D(X)$, intuitively " $X$ plus diagonals", can be defined by

$$
V(D)=V(X) \text { and } E(D)=E(X) \cup\{v w \mid \partial(v, w)=\delta\}
$$

where $v w$ is the edge $\{v, w\}$. Obviously, $\operatorname{Aut}(X) \leqslant \operatorname{Aut}(D)$, so that $D$ is transitive if $X$ is.

A $t$-arc of $X$ is a sequence $\left(v_{0}, v_{1}, \ldots, v_{t}\right)$ of vertices of $X$ such that $v_{i-1} v_{i} \in$ $E(X)$ for $1 \leqslant i \leqslant t$ and $v_{i-1} \neq v_{i+1}$, for $1 \leqslant i<t$. The arc-transitivity of $X$ is defined to be the maximum value of $t$ such that $\operatorname{Aut}(X)$ acts transitively on the $t$-arcs of $X$. A discussion of arc-transitivity may be found in Biggs [2] .

Let $\pi$ be a partition of $V(X)$ into possiblyempty subsets $V_{1}$ and $V_{2}$. The operation of switching $X$ about $\pi$ produces a graph $Y$, where

$$
V(Y)=V(X), \text { and }
$$

$E(Y)=\left\{v w \in E(X) \mid v, w \in V_{1}\right.$ or $\left.v, w \in V_{2}\right\} \cup\left\{v w \notin E(X) \mid v \in V_{1}\right.$ and $\left.w \in V_{2}\right\}$. 


\begin{tabular}{|c|c|c|c|c|c|c|c|c|c|c|}
\hline & & & & & gree & & & & & \\
\hline order & 0 & 1 & 2 & 3 & 4 & 5 & 6 & 7 & 8 & total \\
\hline 1 & 1 & & & & & & & & & 1 \\
\hline 2 & 0 & 1 & & & & & & & & 1 \\
\hline 3 & 0 & 0 & 1 & & & & & & & 1 \\
\hline 4 & 0 & 0 & 1 & 1 & & & & & & 2 \\
\hline 5 & 0 & 0 & 1 & 0 & 1 & & & & & 2 \\
\hline 6 & 0 & 0 & 1 & 2 & 1 & 1 & & & & 5 \\
\hline 7 & 0 & 0 & 1 & 0 & 1 & 0 & 1 & & & 3 \\
\hline 8 & 0 & 0 & 1 & 2 & 3 & 2 & 1 & 1 & & 10 \\
\hline 9 & 0 & 0 & 1 & 0 & 3 & 0 & 2 & 0 & 1 & 7 \\
\hline 10 & 0 & 0 & 1 & 3 & 3 & 4 & 3 & 2 & 1 & 18 \\
\hline 11 & 0 & 0 & 1 & 0 & 2 & 0 & 2 & 0 & 1 & 7 \\
\hline 12 & 0 & 0 & 1 & 4 & 10 & 12 & 13 & 11 & 7 & 64 \\
\hline 13 & 0 & 0 & 1 & 0 & 3 & 0 & 4 & 0 & 3 & 13 \\
\hline 14 & 0 & 0 & 1 & 3 & 5 & 6 & 8 & 9 & 6 & 51 \\
\hline 15 & 0 & 0 & 1 & 0 & 7 & 0 & 12 & 0 & 12 & 44 \\
\hline 16 & 0 & 0 & 1 & 4 & 13 & 25 & 39 & 47 & 48 & 272 \\
\hline 17 & 0 & 0 & 1 & 0 & 4 & 0 & 7 & 0 & 10 & 35 \\
\hline 18 & 0 & 0 & 1 & 5 & 12 & 23 & 36 & 45 & 53 & 365 \\
\hline 19 & 0 & 0 & 1 & 0 & 4 & 0 & 10 & 0 & 14 & 59 \\
\hline
\end{tabular}

TABLE 2. Number of connected transitive graphs

Switching provides an equivalence relation on the set of all graphs. Each equivalence class contains at most one transitive graph of odd order (see [4]). However, if the number of vertices is even, an equivalence class may contain many transitive graphs. Details appear in Section 5.

An important object associated with switching is the switching graph. The switching graph of $X$, denoted $\operatorname{Sw}(X)$, is defined to have vertex set $\{v \mid v \in V(X)\} \cup$ $\left\{v^{\prime} \mid v \in V(X)\right\}$ and edge set

$$
E(\operatorname{Sw}(X))=E(X) \cup\left\{v^{\prime} w^{\prime} \mid v w \in E(X)\right\} \cup\left\{v w^{\prime} \mid v \neq w \text { and } v w \notin E(X)\right\} .
$$

If $X$ has $n$ vertices, $\mathrm{Sw}(X)$ has $2 n$ vertices and is regular with degree $n-1$. Godsil [3] has shown that two graphs $X$ and $Y$ are equivalent under switching if and only if their switching graphs are isomorphic. Clearly, $\operatorname{Sw}(X)$ is transitive whenever $X$ is, but it is possible for a transitive switching graph to be not derived from any transitive graph. The only example in our catalogue is the icosahedron L37.

A large number of transitive graphs can be obtained from groups by means of the Cayley graph construction. Let $G$ be a group, and $H$ be a subset of $G$ such that 
(i) $H$ does not contain the identity, and

(ii) $g \in H \Rightarrow g^{-1} \in H$ for all $g \in G$.

The Cayley graph of $G$ with connection set $H$ is the graph $X=X(G, H)$ with

$$
V(X)=G \text { and } E(X)=\{\{g, g h\} \mid g \in G, h \in H\} .
$$

$X$ is a transitive graph on which $G$ acts (by left multiplication) as a regular subgroup of $\operatorname{Aut}(X)$. If in fact $\operatorname{Aut}(X) \cong G, X$ is called a graphical regular representation (GRR) of $G$. In Section 5 we will give some examples of transitive graphs which are not Cayley graphs for any group.

Let $X$ and $Y$ be any graphs. We will define three products of $X$ and $Y$, all of which have vertex set $V(X) \times V(Y)$.

(a) The cartesian product $X \times Y$ has

$$
\begin{aligned}
& E(X \times Y)=\left\{\left(x_{1}, y_{1}\right)\left(x_{2}, y_{2}\right) \mid x_{1}=x_{2} \text { and } y_{1} y_{2} \in E(Y),\right. \text { or } \\
& \left.\qquad y_{1}=y_{2} \text { and } x_{1} x_{2} \in E(X)\right\} .
\end{aligned}
$$

(b) The tensor product (conjunction) $X * Y$ has

$$
E(X * Y)=\left\{\left(x_{1}, y_{1}\right)\left(x_{2}, y_{2}\right) \mid x_{1} x_{2} \in E(X) \text { and } y_{1} y_{2} \in E(Y)\right\} \text {. }
$$

(c) The lexicographic product (composition) $X[Y]$ has

$$
E(X[Y])=\left\{\left(x_{1}, y_{1}\right)\left(x_{2}, y_{2}\right) \mid x_{1} x_{2} \in E(X) \text {, or } x_{1}=x_{2} \text { and } y_{1} y_{2} \in E(Y)\right\} \text {. }
$$

Note that $X \times Y \cong Y \times X$ and $X * Y \cong Y * X$, but that $X[Y] \not \equiv Y X]$, in general.

3. Groups of Order $n, 5 \leqslant n \leqslant 19$. In this section we give a list of all groups with orders 5 through 19, with their elements and some statistics. This information will be needed in Section 4, where we give representations of transitive graphs as Cayley graphs. Within each order, the abelian groups precede the nonabelian ones. Each group is generated by those elements $A, B, C, \ldots$ which appear in the list of relators. Inverses $A^{-1}, B^{-1}, C^{-1}, \ldots$ are abbreviated to $Z, Y, X, \ldots$, respectively. A number following a letter raises that letter to the given power. A number which begins a word raises that whole word to the given power.

For example, BA3 means $B A^{3}, 3 Z 2 C$ means $\left(A^{-2} C\right)^{3}$.

(a) List of Relators: For example, the relators $4 \mathrm{~A} 4 \mathrm{~B} \mathrm{ZYAB}$ show that group $16-3$ is $\left\langle A, B \mid A^{4}=B^{4}=A^{-1} B^{-1} A B=1\right\rangle$.

(b) Statistics:

$$
\begin{aligned}
\text { INV } & =\text { number of involutions, } \\
\text { EXP } & =\text { exponent }, \\
\text { CNTR } & =\text { order of centre, } \\
\text { COMM } & =\text { order of commutator subgroup, } \\
\text { NSQ } & =\text { number of squares (including } 1 \text { ). }
\end{aligned}
$$

(c) List of Elements: Each element is given as a word of shortest possible length in the generators and their inverses. Those words before the comma are involu- 
tions, while those after the comma are one element of each pair $\left\{g, g^{-1}\right\}$, where the order of $\mathrm{g}$ is greater than 2 . If there is no comma, each element is an involution.

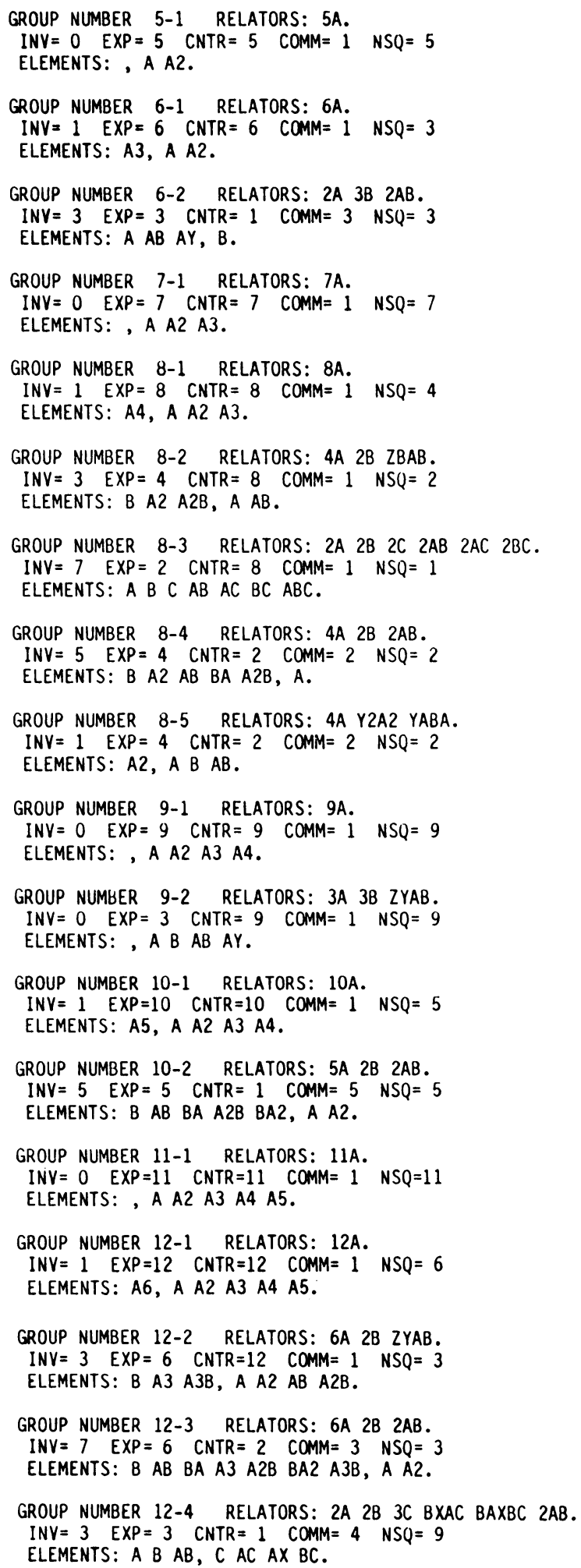


GROUP NUMBER 12-5 RELATORS: 6A Y2A3 AYAB. $I N V=1 \quad E X P=6 \quad C N T R=2 \quad C O M M=3 \quad N S U=4$ ELEMENTS: B2, A B A2 AB BA.

GROUP NUMBER 13-1 RELATORS: 13A.

INV $=0 \quad$ EXP $=13 \quad$ CNTR $=13 \quad$ COMM $=1 \quad N S Q=13$

ELEMENTS: , A A2 A3 A4 A5 A6.

GROUP NUMBER 14-1 RELATORS: 14A.

INV $=1 \quad$ EXP $=14 \quad$ CNTR $=14 \quad$ COMM $=1 \quad N S Q=7$

ELEMENTS: A7, A A2 A3 A4 A5 A6.

GROUP NUMBER 14-2 RELATORS: 7A 2B 2AB.

$I N V=7 \quad E X P=7 \quad C N T R=1 \quad C O M M=7 \quad N S Q=7$

ELEMENTS: $B \quad A B$ BA A2B BA2 A3B BA3, A A2 A3.

GROUP NUMBER 15-1 RELATORS: 15 A.

INV $=0 \quad$ EXP $=15 \quad$ CNTR $=15 \quad$ COMM $=1 \quad N S Q=15$

ELEMENTS: , A A2 A3 A4 A5 A6 A7.

GROUP NUMBER 16-1 RELATORS: 16A.

INV $=1 \quad$ EXP $=16 \quad$ CNTR=16 COMM $=1 \quad N S Q=8$

ELEMENTS: A8, A A2 A3 A4 A5 A6 A7.

GROUP NUMBER 16-2 RELATORS: 8 A $2 B$ ZYAB.

INV $=3 \quad$ EXP $=8 \quad$ CNTR $=16 \quad$ COMM $=1 \quad N S U=4$

ELEMENTS: $B$ A4 A4B, A A2 AB A3 A2B A3B.

GROUP NUMBER 16-3 RELATORS: 4A 4B ZYAB.

INV $=3 \quad$ EXP $=4 \quad$ CNTR $=16 \quad$ COMM $=1 \quad$ NSQ $=4$

ELEMENTS: $A 2$ B2 A2B2, A B AB AY A2B AB2.

GROUP NUMBER 16-4 RELATORS: $4 A$ 2B 2C ZBAB 2BC ZCAC.

INV $=7 \quad$ EXP $=4 \quad$ CNTR $=16 \quad$ COMM $=1 \quad N S Q=2$

ELEMENTS: $B C A 2 B C A 2 B A 2 C A 2 B C, A A B A C A B C$.

GROUP NUMBER 16-5 RELATORS: $2 A$ 2B $2 C \quad 202 A B \quad 2 A C \quad 2 A D 2 B C 2 B D 2 C D$. INV $=15 \quad$ EXP $=2 \quad$ CNTR $=16 \quad$ COMM $=1 \quad N S Q=1$

ELEMENTS: $A B C D A B$ AC $A D B C B D C D$ ABC ABD ACD BCD ABCD.

GROUP NUMBER 16-6 RELATORS: $4 A 2 B$ 2C $2 A B$ ZCAC $2 C B$.

INV $=11 \quad$ EXP $=4 \quad$ CNTR $=4 \quad$ COMM $=2 \quad N S Q=2$

ELEMENTS: $B C A 2 A B$ BA $B C$ A2B A2C ABC BAC A2BC, $A$ AC.

GROUP NUMBER 16-7 RELATORS: 4A 2B X2A2 AXAC XBCB ZBAB.

INV $=3 \quad$ EXP $=4 \quad$ CNTR $=4 \quad$ COMM $=2 \quad N S Q=2$

ELEMENTS: $B$ A2 A2B, $A C A B A C B C A B C$.

GROUP NUMBER 16-8 RELATORS: $4 A$ 2B X2A2 AXAC 2BC ZBAB.

INV $=7 \quad$ EXP $=4 \quad$ CNTR $=4 \quad$ COMM $=2 \quad N S Q=2$

ELEMENTS: $B A 2 B C B X A 2 B A B C A B X, A C A B A C$.

GROUP NUMBER 16-9 RELATORS: 4A 2B X2B YAXAC ZBAB.

INV $=7 \quad E X P=4 \quad C N T R=4 \quad C O M M=2 \quad N S Q=3$

ELEMENTS: $B$ A2 AC AX CA ZC A2B, A C AB A2C.

GROUP NUMBER 16-10 RELATORS: $4 A$ 4B AYAB.

INV $=3 \quad E X P=4 \quad C N T R=4 \quad C O M M=2 \quad N S O=3$

ELEMENTS: $A 2$ B2 A2B2, A B AB BA A2B AB2.

GROUP NUMBER 16-11 RELATORS: 8A 2B 25BAB.

INV $=3 \quad E X P=8 \quad C N T R=4 \quad C O M M=2 \quad N S Q=4$

ELEMENTS: $B$ ABZ A4, $A$ A2 AB BA A3 A2B:

GROUP NUMBER 16-12 RELATORS: $8 A$ 2B $2 A B$.

INV $=9 \quad$ EXP $=8 \quad$ CNTR $=2 \quad$ COMM $=4 \quad N S Q=4$

ELEMENTS: $B$ AB BA A2B BA2 A4 A3B BA3 A4B, A A2 A3.

GROUP NUMBER 16-13 RELATORS: 8A 2B 23BAB.

INV $=5 \quad$ EXP $=8 \quad$ CNTR $=2 \quad$ COMM $=4 \quad N S Q=4$

ELEMENTS: $B$ A2B ABA ABZ A4, $A$ A2 $A B$ BA $A 3$.

GROUP NUMBER 16-14 RELATORS: 8A Y2A4 AYAB.

INV $=1 \quad E X P=8 \quad$ CNTR $=2 \quad$ COMM $=4 \quad N S Q=4$

ELEMENTS: $B 2, A B$ A2 AB BA A3 A2B. 


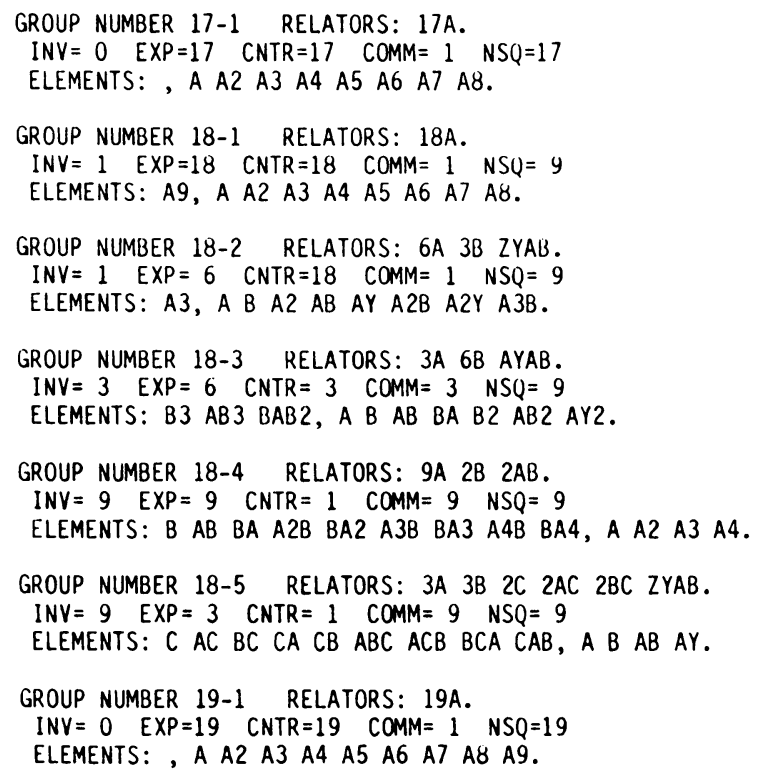

4. Transitive Graphs of Order $n, 2 \leqslant n \leqslant 19$. The catalogue in this section contains data on every transitive graph with $n$ vertices and degree $d$, for $2 \leqslant n \leqslant 9$ (any $d$ ) and $10 \leqslant n \leqslant 19$ (for $d \leqslant(n-1) / 2$ ). Those with the remaining degrees can be obtained by complementation. In describing the information presented for some particular graph, we will refer to the graph as $X$, and use $n$ and $d$ to denote its order and degree, respectively.

(a) Set Notation: A set of positive integers can be written as an octal integer by putting bit $i$ equal to 1 if and only if $i$ is in the set. The bits are numbered from 1 , starting at the right hand (low order) end. For example, 251 (octal) is 10101001 (binary) and so represents the set $\{1,4,6,8\}$.

(b) First Line of Data: The first item in this line is the name of $X$, for example L20 or P16. The letter indicates the order of $X$ (A for 1, B for 2, etc.), and the numbers are allotted sequentially within each order. Care must be taken to avoid confusing names like $K 3$ with the commonly accepted notations [1] for special graphs, for example $K_{3}, C_{5}, K_{3,4}$. The latter notations will be used in this description of the catalogue, but never in the catalogue itself.

We now describe the other pieces of information which may occur on the first line.

(i) DEG: degree of $X$.

(ii) F: flags associated with $X$. Each flag is a single letter whose presence indicates a special property. If no flags apply, the $\mathrm{F}$ is omitted. The flags used are listed below.

$\mathrm{X}=$ disconnected.

$\mathrm{N}=$ not a Cayley graph.

$\mathrm{T}=$ distance transitive.

$\mathrm{R}=\quad$ distance regular but not distance transitive (only case is P84).

$\mathrm{V}=\operatorname{Aut}(X)$ acts primitively on $V(X)$. 
$\mathrm{I}=\quad \operatorname{Aut}(X)$ satisfies this condition: For any $v, w \in V(X)$ there is $\alpha \in \operatorname{Aut}(X)$ such that $v^{\alpha}=w$ and $w^{\alpha}=v$.

$\mathrm{A}=$ antipodal.

$\mathrm{S}=$ self-complementary.

$\mathrm{P}=$ planar.

(iii) AUT: order of $\operatorname{Aut}_{1}(X)$.

(iv) P: partitions $\partial(X)$ and $\alpha(X)$. Each digit or letter gives the size of one cell of a partition $\pi$ of $V(X)$. Letters are used for cell sizes over $9 ; A$ for $10, B$ for 11 , etc.

Case 1: If $n=2$ or $X$ is not a GRR, then $\pi$ is $\alpha(X)$. The cells of $\alpha(X)$ are grouped by commas into the cells of $\partial(X)$. For example, $\mathrm{P}=(1,4,24,1)$ indicates that $\alpha(X)$ has one 4-cell at distance 1 from vertex 1 , a 2 -cell and a 4-cell at distance 2 , and a single 1 -cell at distance 3 . If $X$ is disconnected, only vertices in the component containing vertex 1 are included; the presence of additional components is indicated by a " + " sign.

Case 2: If $n \neq 2$ and $X$ is a GRR, then $\pi$ is $\partial(X)$. To avoid confusion with Case 1 , the cells are separated by slashes. For example, $\mathrm{P}=(1 / 6 / 8 / 1)$ indicates 6 vertices at distance 1 from vertex 1,8 vertices at distance 2 , and 1 vertex at distance 3 .

(v) GIR: girth of $X$, unless $X$ is acyclic.

(vi) $\mathrm{CN}$ : chromatic numbers of $X$ and $\bar{X}$, respectively.

(vii) $\mathrm{T}$ : $\operatorname{arc}$-transitivity of $X$, unless $\operatorname{Aut}(X)$ is not transitive on 1-arcs, or $d=0$, or $d=2$.

(viii) Any other text on the first line indicates a common name for $X$, for example "PETERSEN GRAPH".

(c) Adjacency Matrix (omitted if $d=0$ ).

$$
A=a_{2} a_{3} a_{4} a_{5}, a_{6} \cdots a_{n} .
$$

Each $a_{i}$ is an octal representation (see part (a)) of the set of vertices preceding vertex $i$ which are adjacent to $i$. Note that $a_{1}$ is omitted. The labelling of the vertices of $X$ is consistent with the partition $\mathrm{P}$ described above. For example, if $\mathrm{P}=$ $(1,4,24,1), \alpha(X)$ is $\{1|2,3,4,5| 6,7|8,9,10,11| 12\}$ and $\partial(X)$ is $\{1|2,3,4,5| 6$, $7,8,9,10,11 \mid 12\}$.

Example: If $A=116$, we have 2 adjacent to 1,3 adjacent to 1 and 4 adjacent to 2 and 3.

(d) Eigenvalues of Adjacency Matrix (omitted if $X$ is disconnected).

$$
E=m_{1} \lambda_{1} m_{2} \lambda_{2} \cdots
$$

Each field gives one eigenvalue of the adjacency matrix of $X$. If the eigenvalue has multiplicity other than one, this multiplicity is written immediately before the eigenvalue, using an intervening " + " for nonnegative eigenvalues. If the eigenvalues for $X$ are $\lambda_{1} \leqslant \lambda_{2} \leqslant \cdots \leqslant \lambda_{n}$, those for $\bar{X}$ are $-\lambda_{n-1}-1 \leqslant-\lambda_{n-2}-1 \leqslant \cdots \leqslant-\lambda_{1}$ $-1 \leqslant n-d-1$. 
Example: $E=-43-\cdot 43912+01 \cdot 34175$

-the eigenvalues are -0.4391 ( 3 times), 0 (twice) and $-4,1 \cdot 3417$,

5 (once each).

(e) Independent Sets and Cliques (omitted if $X$ is disconnected).

$$
K=\left(\alpha_{3} \alpha_{4}, \ldots, \beta_{3} \beta_{4} \cdots\right) .
$$

$\alpha_{i}$ is the number of independent sets of size $i$ in $X$, i.e. cliques of size $i$ in $\bar{X}$, which include vertex 1 .

$\beta_{i}$ is the number of cliques of size $i$ in $X$ which include vertex 1 .

Those numbers before the comma are $\alpha$ 's; those after the comma are $\beta$ 's. The total number of independent sets or cliques of size $i$ in $X$ is $n \alpha_{i} / i$ or $n \beta_{i} / i$, respectively.

Example: $K=(, 41)$. $X$ has no independent sets of size 3 or greater. Vertex 1 is contained in 4 triangles and 1 clique of size 4.

(f) Representations of $X$. The data provided about $X$ contain a number of descriptors expressing $X$ as a product etc. In explaining each descriptor type, $Y$ and $Z$ stand for the names of transitive graphs in the catalogue. As before, $n$ and $d$ are the order and degree of $X$, respectively. The variable $i$ indicates a positive integer.

(i) $\quad-Y: \quad$ complement of $Y$, unless $X$ is self-complementary.

(ii) $\quad i[Y]: \quad X$ is the disjoint union of $i$ copies of $Y(i>1)$, unless $d \leqslant 1$.

(iii) $\quad L(Y): \quad$ linegraph of $Y$, unless $d \leqslant 2$.

(iv) $\quad-L(Y)$ : complement of $L(Y)$, unless $d \leqslant 2$ or $X$ is complete.

(v) $\quad S W(Y)$ : switching graph of $Y$.

(vi) $\quad S W(Y+):$ switching graph of the disjoint union $Y \cup K_{1}$, unless $Y$ is complete or empty. The only example is L37. All switching graphs in the catalogue are either type (v) or type (vi).

(vii) $\quad D(Y): \quad Y$ plus diagonals (see Section 2 ), provided $Y$ has diameter at least 3 and is connected.

(viii) $\quad-D(Y)$ : complement of $D(Y)$. This notation is omitted if $Y$ is bipartite and has diameter 3 . In that case $-D(Y)$ is the disjoint union of two cliques. $Y$ is connected with diameter $\geqslant 3$.

(ix) $\quad$ Wi(Y): generalized linegraph of subdivision graph $(1 \leqslant i \leqslant 9)$. Form a multigraph from $Y$ by replacing each edge by $i$ parallel edges. Then subdivide each edge with a new vertex and take the linegraph of the result. Omitted if $Y$ has degree $\leqslant 1$, or $X$ has degree 2 .

Every linegraph in the catalogue is of type (iii) or type (ix) except these:

$$
\begin{array}{ll}
L\left(K_{1, m}\right)=K_{m} & (2 \leqslant m \leqslant 19), \\
L\left(K_{3, m}\right)=K_{3} \times K_{m} & (4 \leqslant m \leqslant 6) .
\end{array}
$$

(x) $\quad-W i(Y)$ : complement of $W i(Y)(1 \leqslant i \leqslant 9)$, unless $Y$ has degree $\leqslant 1$, or $W i(Y)$ has degree 2. 
(xi) $\quad Y[Z]: \quad$ lexicographic product of $Y$ around $Z$, unless $d \leqslant 1$. If $Y$ is empty (i.e. $\bar{Y}$ is complete), the notation (ii) is used instead.

(xii) $\quad Y \times Z: \quad$ cartesian product of $Y$ and $Z$, unless either $Y$ or $Z$ is empty.

(xiii) $\quad-Y \times Z:$ complement of $Y \times Z$, unless $X$ is complete.

(xiv) $\quad Y * Z: \quad$ tensor product of $Y$ and $Z$, unless $d \leqslant 1$.

(xv) $\quad-Y * Z$ : complement of $Y * Z$, unless $X$ is either empty or complete.

(xvi) $\quad i / m: \quad X$ is the Cayley graph $X(G, H)$, where $G$ is the $i$ th group of order $n$, and the connection set $H$ is specified by the octal number $m$ (see (a)). The groups and their elements are numbered in the order they are listed in Section 3; an element and its inverse have the same ordinal. $H$ is not canonical in any sense.

Example: If $n=16$, the notation $3 / 123$ represents $X(G, H)$, where $G$ is group 16-3 and $H$ is $\left\{A^{2}, B^{2}, B^{ \pm 1},\left(A B^{-1}\right)^{ \pm 1}\right\}$.

Cayley graph representation is only given if $2 \leqslant d \leqslant$ $(n-1) / 2$.

Orders two through fifteen are presented here. Sixteen through nineteen appear in the microfiche supplement accompanying this issue.

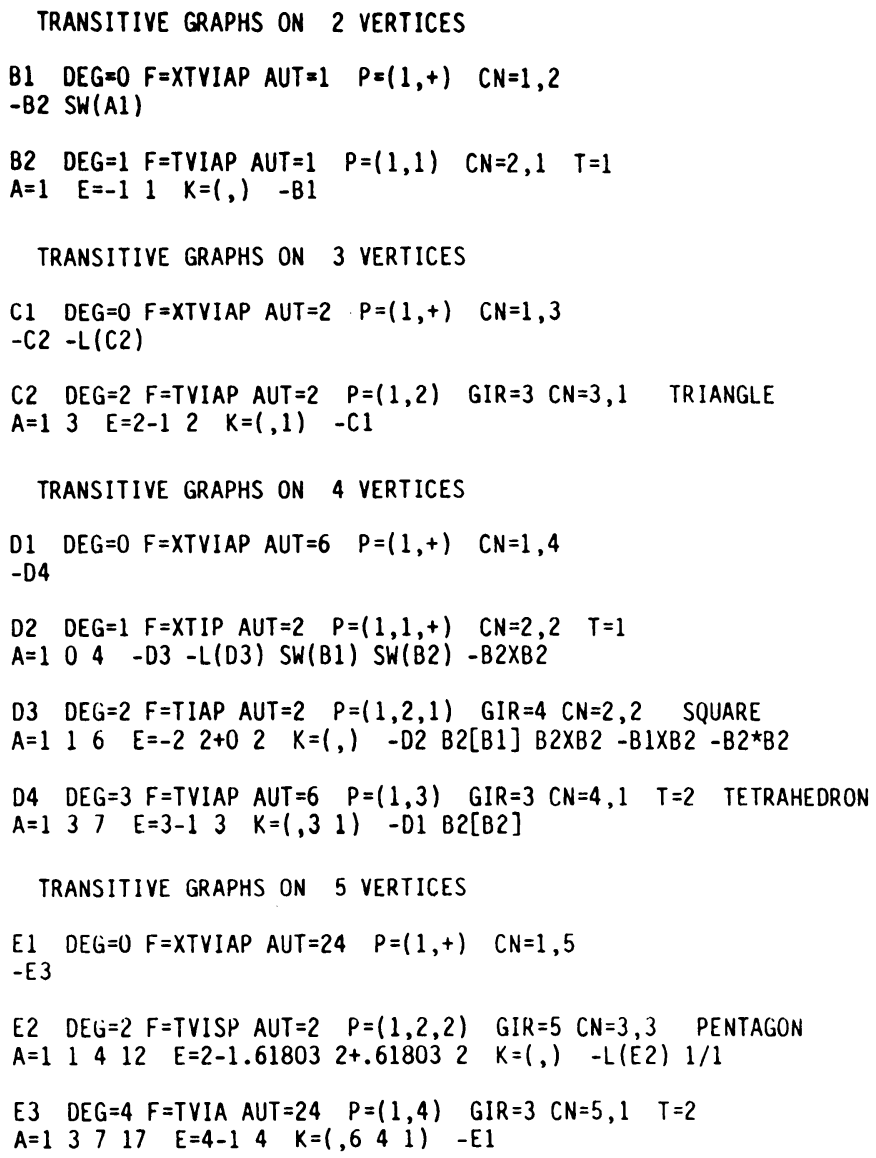


TRANSITIVE GRAPHS ON 6 VERTICES

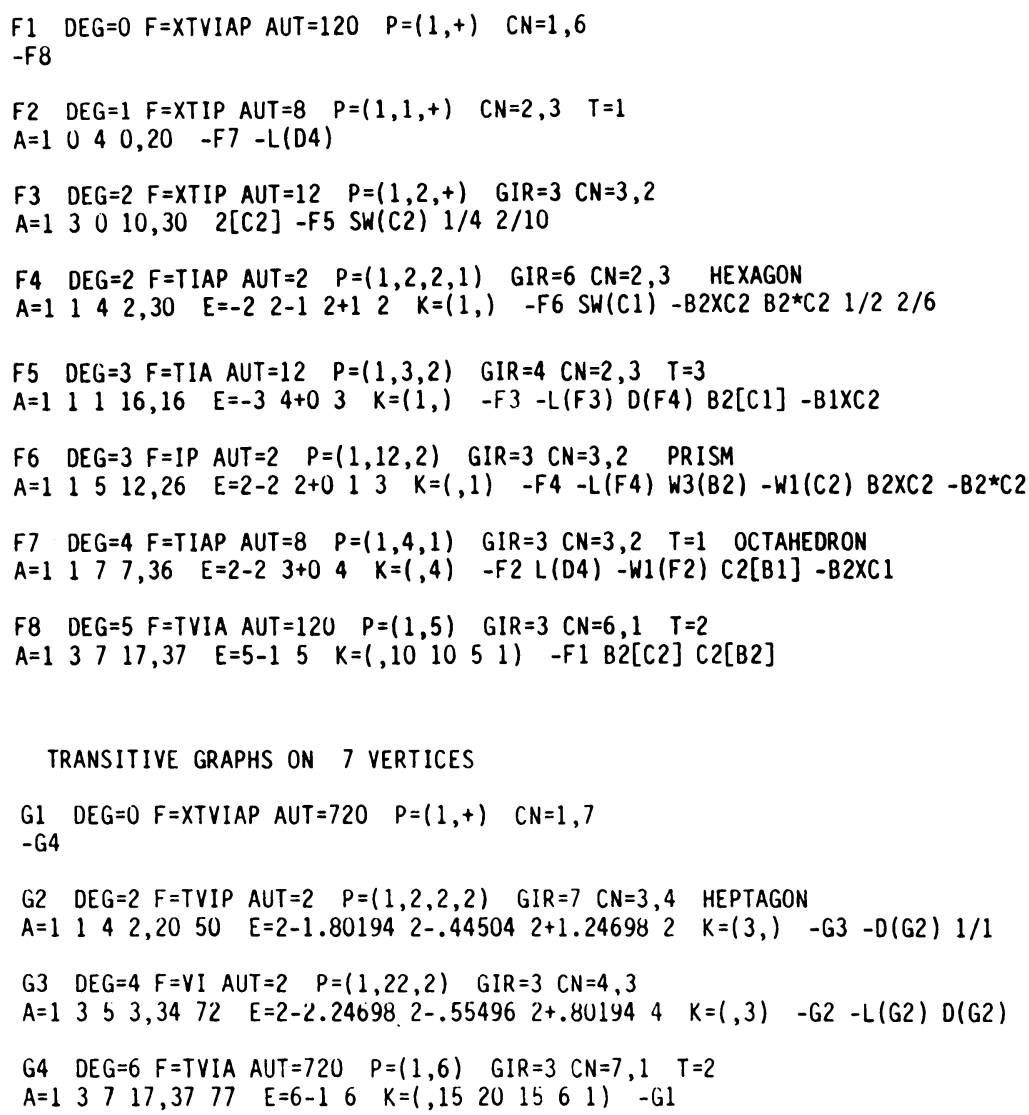


TRANSITIVE GRAPHS ONI 8 VERTICES (CONTD)

H10 DEG=4 $\quad F=I P \quad A U T=2 \quad P=(1,22,12) \quad G I R=3 \quad C N=4,3 \quad$ ANTIPRISM

$A=1 \quad 1 \quad 5 \quad 13,6 \quad 54 \quad 162 \quad E=2-2 \quad 2-1.41421002+1.41421 \quad 4 \quad K=(, 3) \quad-H 6 \quad-D(H 4)$

H11 $D E G=5 \quad F=1 \quad A U T=16 \quad P=(1,14,2) \quad G I R=3 \quad C N=4,2$

$A=1 \quad 3 \quad 3 \quad 7,1374 \quad 174 \quad E=-3 \quad 4-1 \quad 2+15 \quad K=(, 6 \quad 2) \quad-H 3 \quad-L(H 3)-W 2$ (D2) B2[D2] D3[B2] $-B 1 \times D 3-B 2 \times D 2-B 2 \star D 3$

H12 DEG $=5 \quad F=1 \quad A U T=2 \quad P=(1,122,2) \quad G I R=3 \quad C N=4,2$

$A=1 \quad 1 \quad 5 \quad 13,27 \quad 56 \quad 136 \quad E=2-2.41421 \quad 2-1 \quad 2+.41421 \quad 1 \quad 5 \quad K=\left(\begin{array}{llllll}.6 & 1\end{array}\right) \quad-H 4 \quad-L(H 4)$ $-W 1(03)$

H13 DEG=6 F=TIA AUT $=48 \quad P=(1,6,1) \quad G I R=3 \quad C N=4,2 \quad T=1$

$A=1 \quad 1 \quad 7 \quad 7,37 \quad 37 \quad 176 \quad E=3-2 \quad 4+0 \quad 6 \quad K=(, 128) \quad-H 2 \quad-W 1(H 2) \quad B 2[D 3] \quad D 4[B 1]-B 1 \times D 2$ $-B 2 \times D 1-B 2 * D 2$

H14 DEG=7 F=TVIA AUT $=5040 \quad P=(1,7) \quad G I R=3 \quad C N=8,1 \quad T=2$

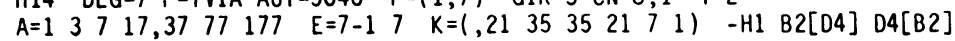

TRANSITIVE GRAPHS ON 9 VERTICES

I1 DEG $=0 \quad F=X T V I A P \quad A U T=40320 \quad P=(1,+) \quad C N=1,9$

$-19$

12 DEG $=2 \quad F=X T I P$ AUT $=144 \quad P=(1,2,+) \quad G I R=3 \quad C N=3,3$

$A=130010,03040240 \quad 3[C 2]-17 \quad 1 / 4 \quad 2 / 4$

I3 DEG $=2 \quad F=T I P$ AUT $=2 \quad P=(1,2,2,2,2) \quad G I R=9 \quad C N=3,5 \quad$ NONAGON

$A=1 \quad 1 \quad 4 \quad 2,2010 \quad 100240 \quad E=2-1.87939 \quad 2-1 \quad 2+.34730 \quad 2+1.532092 \quad K=\left(\begin{array}{lllll}10 & 4,1\end{array}-18\right.$ $1 / 10$

I4 $D E G=4 \quad F=T V I S \quad A U T=8 \quad P=(1,4,4) \quad G I R=3 \quad C N=3,3 \quad T=1$

$A=1 \quad 3 \quad 1 \quad 11,24 \quad 12 \quad 154 \quad 162 \quad E=4-2 \quad 4+1 \quad 4 \quad K=(2,2) \quad L(F 5)-L(F 5) \quad C 2 \times C 2-C 2 X C 2 \quad C 2 * C 2$ $-\mathrm{C} 2 \star \mathrm{C} 2 \mathrm{2} / 12$

I5 $D E G=4 \quad F=I \quad A U T=2 \quad P=(1,22,22) \quad G I R=3 \quad C N=3,3$

$A=1 \quad 3 \quad 1 \quad 1,3432 \quad 124 \quad 252 \quad E=2-2.87939 \quad 2-.65270 \quad 2+.53209 \quad 2+1 \quad 4 \quad K=(3,1) \quad-16$ $-D(13) 1 / 14$

I6 $D E G=4 \quad F=1 \quad A U T=2 \quad P=(1,22,22) \quad G I R=3 \quad C N=3,3$

$A=1 \quad 1 \quad 3 \quad 15,24 \quad 12 \quad 144 \quad 342 \quad E=2-2 \quad 2-1.53209 \quad 2-.34730 \quad 2+1.87939 \quad 4 \quad K=(1,3) \quad-15$ D(I3) $1 / 11$

$17 \quad D E G=6 \quad F=T I A \quad A U T=144 \quad P=(1,6,2) \quad G I R=3 \quad C N=3,3 \quad T=1$

$A=1 \quad 1 \quad 1 \quad 17,17 \quad 17 \quad 176 \quad 176 \quad E=2-3 \quad 6+0 \quad 6 \quad K=(1,9) \quad-I 2 \quad-L(I 2) \quad C 2[C 1]-C 1 X C 2$

$18 \quad D E G=6 \quad F=1 \quad A U T=2 \quad P=(1,222,2) \quad G I R=3 \quad C N=5,3$

$A=1 \quad 3 \quad 5 \quad 13,27 \quad 17 \quad 174 \quad 372 \quad E=2-2.53209 \quad 2-1.34730 \quad 2+0 \quad 2+.87939 \quad 6 \quad K=(, 104) \quad-I 3$ $-L(13)$

19 $D E G=8 \quad F=T V I A \quad A U T=40320 \quad P=(1,8) \quad G I R=3 \quad C N=9,1 \quad T=2$

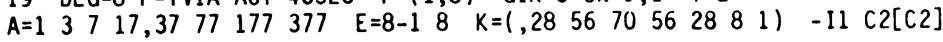

TRANSITIVE GRAPHS ON 10 VERTICES

J1 $D E G=0 \quad F=X T$ TVIAP $A U T=362880 \quad P=(1,+) \quad C N=1,10$

J2 DEG=1 $F=X T I P \quad A U T=384 \quad P=(1,1,+) \quad C N=2,5 \quad T=1$

$A=104 \quad 0,2001000400$

J3 $D E G=2 \quad F=X T I P \quad A U T=20 \quad P=(1,2,2,+) \quad G I R=5 \quad C N=3,6$

$A=1 \quad 14 \quad 12,040 \quad 0300240 \quad$ 2[E2] $1 / 4 \quad 2 / 40$

$J 4$ DEG $=2$ F $=$ TIAP AUT $=2 \quad P=(1,2,2,2,2,1) \quad G I R=10 \quad C N=2,5 \quad$ POLYGON

$A=1 \quad 142,2010 \quad 10040 \quad 600 \quad E=-2 \quad 2-1.61803 \quad 2-.61803 \quad 2+.61803 \quad 2+1.618032$

$K=\left(\begin{array}{lll}15 & 10 & 1,\end{array}\right) \quad B 2 \star E 2 \quad 1 / 10 \quad 2 / 24$

J5 $D E G=3 \quad F=I \quad A U T=2 \quad P=(1,12,22,2) \quad G I R=4 \quad C N=2,5$

$A=1 \quad 1 \quad 1 \quad 12,6 \quad 4 \quad 10 \quad 320340 \quad E=-3 \quad 2-1.61803 \quad 2-.61803 \quad 2+.61803 \quad 2+1.618033$

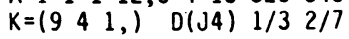


TRANSITIVE GRAPHS ON 10 VERTICES (CONTD)

J6 $D E G=3 \quad F=I P \quad A U T=2 \quad P=(1,12,22,2) \quad G I R=4 \quad C N=3,5 \quad P R I S M$

$A=1 \quad 1 \quad 1 \quad 12,6 \quad 10 \quad 104 \quad 240 \quad 520 \quad E=2-2.61803 \quad 2-.61803 \quad 2-.38197 \quad 1 \quad 2+1.61803 \quad 3$

$K=(94$,$) \quad B2XE2 1 / 21 \quad 2 / 41$

J7 DEG=3 $F=$ NTVI AUT $=12 \quad P=(1,3,6) \quad G I R=5 \quad C N=3,5 \quad T=3 \quad$ PETERSEN GRAPH

$A=11110,2210102144224 \quad E=4-2 \quad 5+13 \quad K=(9 \quad 2) \quad-,L(E 3)$

J8 $\quad D E G=4 \quad F=X T I \quad A U T=2880 \quad P=(1,4,+) \quad G I R=3 \quad C N=5,2 \quad T=2$

$A=13717,040140340740$ 2[E3] SW(E3) $1 / 24 \quad 2 / 140$

J9 $D E G=4 \quad F=I \quad A U T=32 \quad P=(1,4,14) \quad G I R=4 \quad C N=3,5 \quad T=1$

$A=11111,3630106106 \quad 630 \quad E=2-3.23607 \quad 5+0 \quad 2+1.23607 \quad 4 \quad K=\left(\begin{array}{llll}6 & 2,) \quad E 2[B 1] & 1 / 14\end{array}\right.$ $2 / 130$

JIU DEG=4 F=TIA AUT $=24 \quad P=(1,4,4,1) \quad G I R=4 \quad C N=2,5 \quad T=2$

$A=1 \quad 1 \quad 1 \quad 1,34 \quad 32 \quad 26 \quad 16740 \quad E=-4 \quad 4-1 \quad 4+1 \quad 4 \quad K=\left(\begin{array}{lll}6 & 4 & 1,\end{array}\right) \quad S W(E 1)-W 5(B 2)-B 2 X E 3$

$B 2 \star E 3 \quad 1 / 12 \quad 2 / 33$

J11 $D E G=4 \quad F=I A P \quad A U T=2 \quad P=(1,22,22,1) \quad G I R=3 \quad C N=4,4 \quad$ ANTIPRISM

$A=1 \quad 1 \quad 3 \quad 15,24 \quad 12 \quad 44 \quad 302 \quad 740 \quad E=2-2.23607 \quad 4-1 \quad 0 \quad 2+2.23607 \quad 4 \quad K=(3,3) \quad S W(E 2)$

$-D(J 11)-D(J 6) 1 / 62 / 43$

TRANSITIVE GRAPHS ON 11 VERTICES

K1 DEG $=0 \quad F=X T$ TIAP AUT $=3628800 \quad P=(1,+) \quad C N=1,11$

K2 DEG=2 F=TVIP AUT $=2 \quad P=(1,2,2,2,2,2) \quad G I R=11 \quad C N=3,6 \quad$ POLYGON

$A=1 \quad 1 \quad 4 \quad 2,201010040 \quad 400,1200$

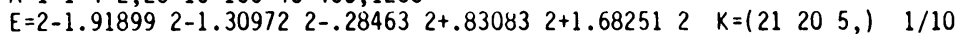

K3 DEG=4 F=VI AUT $=2 \quad P=(1,22,22,2) \quad G I R=3 \quad C N=4,4$

$A=1 \quad 1 \quad 3 \quad 15,24 \quad 12 \quad 102 \quad 44 \quad 640,1700$

$E=2-2.20362 \quad 2-1.594352-.478892-.236482+2.51334 \quad 4 \quad K=(6,3) \quad D(K 2) \quad-D(K 3) \quad 1 / 24$

K4 DEG=4 F=VI AUT $=2 \quad P=(1,22,222) \quad G I R=4 \quad C N=3,6$

$A=1 \quad 1 \quad 1 \quad 1,3432 \quad 104242 \quad 424,1212$

$E=2-3.22871 \quad 2-1.088162+.372792+.546202+1.39788 \quad 4 \quad K=\left(\begin{array}{lll}9 & 4,1\end{array}\right) \quad 1 / 5$

TRANSITIVE GRAPHS ON 12 VERTICES

L1 $D E G=0 \quad F=X T V I A P \quad A U T=39916800 \quad P=(1,+) \quad C N=1,12$

L2 DEG=1 $F=X$ XIIP AUT $=3840 \quad P=(1,1,+) \quad C N=2,6 \quad T=1$

$A=1040,2001000400,02000$

L3 DEG $=2 \quad F=X T I P$ AUT $=2592 \quad P=(1,2,+) \quad G I R=3 \quad C N=3,4$

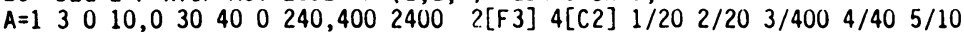

L4 DEG=2 F=XTIP AUT=256 $P=(1,2,1,+) \quad G I R=4 \quad C N=2,6$

$A=1 \quad 1 \quad 6 \quad 0,20020240100,1003000 \quad 3[03] \quad F 2[B 1] \quad B 2 X F 2 \quad 1 / 10 \quad 2 / 5 \quad 3 / 110 \quad 4 / 5 \quad 5 / 4$

L5 DEG=2 F=XTIP AUT $=24 \quad P=(1,2,2,1,+) \quad G I R=6 \quad C N=2,6$

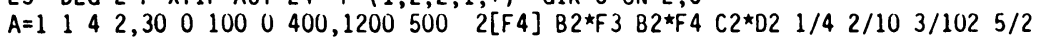

L6 DEG $=2 \quad F=T I A P$ AUT $=2 \quad P=(1,2,2,2,2,2,1) \quad G I R=12 \quad C N=2,6 \quad P O L Y G O N$

$A=1 \quad 1 \quad 4 \quad 2,20 \quad 10 \quad 100 \quad 40 \quad 400,2003000 \quad E=-2 \quad 2-1.73205 \quad 2-1 \quad 2+0 \quad 2+1 \quad 2+1.732052$

$K=\left(\begin{array}{lllll}28 & 35 & 15 & 1,\end{array}\right) \quad 1 / 2 \quad 3 / 120$

L7 DEG=3 F=XTIP AUT $=6912 \quad P=(1,3,+) \quad G I R=3 \quad C N=4,3 \quad T=2$

$A=1370,20060260100,11003100 \quad 3[D 4] \quad F 2[B 2] \quad 1 / 11 \quad 2 / 7 \quad 3 / 34 \quad 4 / 7 \quad 5 / 21$

L8 DEG=3 F=XTI AUT $=864 \quad P=(1,3,2,+) \quad G I R=4 \quad C N=2,6 \quad T=3$

$A=1 \quad 1116,160100100100,16001600 \quad 2[F 5] \quad D 2[C 1] \quad B 2 * F 5 \quad 1 / 5 \quad 2 / 12 \quad 3 / 106 \quad 5 / 3$

L9 $D E G=3 \quad F=X I P \quad A U T=24 \quad P=(1,12,2,+) \quad G I R=3 \quad C N=3,4$

$A=1 \quad 15 \quad 12,260100100200,15001600 \quad$ 2[F6] W3(D2) B2XF3 C2XD2 $1 / 21 \quad 2 / 21 \quad 3 / 401$ $5 / 11$

L10 DEG=3 F=P AUT $=2 \quad P=(1,12,22,22) \quad G I R=3 \quad C N=3,4$

$A=1 \quad 15 \quad 10,42 \quad 102 \quad 240 \quad 120,440 \quad 3020 \quad E=3-2 \quad 3-1 \quad 2+0 \quad 3+2 \quad 3 \quad K=\left(\begin{array}{llll}18 & 10,1\end{array}\right) \quad W 1(D 4)$ $4 / 11$

L11 DEG=3 AUT $=4 \quad P=(1,12,122,12) \quad G I R=4 \quad C N=2,6$

$A=1 \quad 1 \quad 1 \quad 14,104222620,540 \quad 340 \quad E=-3 \quad 2-1.73205 \quad 3-1 \quad 3+1 \quad 2+1.732053$

$K=\left(\begin{array}{lllll}19 & 15 & 5 & 1,\end{array}\right) \quad 3 / 124$ 
TRANSITIVE GRAPHS ON 12 VERTICES (CONTD)

L12 DEG=3 $\quad F=1 \quad$ AUT $=2 \quad P=(1,12,22,22) \quad G I R=4 \quad C N=3,6$

$A=1 \quad 1 \quad 1 \quad 12,6 \quad 104200500,1240520 \quad E=2-2.73205 \quad 3-1 \quad 2+0 \quad 2+.73205 \quad 2+23$

$K=\left(\begin{array}{llll}19 & 16 & 5,\end{array}\right) \quad D(L 6) \quad 1 / 413 / 32$

L13 DEG=3 F=IAP AUT=2 $P=(1,12,22,12,1) \quad G I R=4 \quad C N=2,6 \quad P R I S M$

$A=1 \quad 1 \quad 1 \quad 6,12 \quad 1044300220,1403400 \quad E=-3 \quad 2-2 \quad-1 \quad 4+0 \quad 1 \quad 2+2 \quad 3 \quad K=\left(\begin{array}{llllllll}19 & 16 & 5 & 1,1\end{array}\right)$ B2XF4 B2*F6 2/14 $3 / 122$

L14 DEG=4 F=XTIP AUT $=384 \quad P=(1,4,1,+) \quad G I R=3 \quad C N=3,4 \quad T=1$

$A=1 \quad 1 \quad 7 \quad 7,360100100700,7003600 \quad 2[F 7] \quad L(H 5)-D(L 26) \quad F 3[B 1] 1 / 24 \quad 2 / 120$ $3 / 6005 / 12$

L15 DEG $=4 \quad A U T=4 \quad P=(1,112,122,2) \quad G I R=3 \quad C N=4,3$

$A=1 \quad 1 \quad 5 \quad 15,6 \quad 20 \quad 110 \quad 42 \quad 442,1300 \quad 2700 \quad E=4-2 \quad 2-.73205 \quad 3+0 \quad 2+2.73205 \quad 4 \quad K=\left(\begin{array}{lllll}10,3 & 1\end{array}\right)$ W2(C2) $3 / 56$

L16 DEG $=4$ AUT $=2 \quad P=(1,22,1222) \quad G I R=3 \quad C N=3,4$

$A=1 \quad 1 \quad 1 \quad 11,6 \quad 24 \quad 12 \quad 60450,7021304 \quad E=3-2.56155 \quad 3-1 \quad 2+1 \quad 3+1.56155 \quad 4 \quad K=\left(\begin{array}{llll}12 & 4,1\end{array}\right)$ $4 / 103$

L17 DEG $=4 \quad F=I \quad$ AUT $=2 \quad P=(1,22,122,2) \quad G I R=4 \quad C N=2,6$

$A=1 \quad 1 \quad 1 \quad 1,63432 \quad 22 \quad 14,15401640 \quad E=-4 \quad 2-1.73205 \quad 2-1 \quad 2+0 \quad 2+1 \quad 2+1.73205 \quad 4$

$K=\left(\begin{array}{lllll}13 & 10 & 5 & 1,\end{array}\right) \quad 1 / 50 \quad 3 / 47$

L18 DEG=4 F=1 AUT=4 $P=(1,22,14,2) \quad G I R=3 \quad C N=3,4$

$A=1 \quad 1 \quad 1 \quad 11,6 \quad 24 \quad 22 \quad 114 \quad 212,1440 \quad 2340 \quad E=2-3 \quad 4-1 \quad 0 \quad 2+1 \quad 2+2 \quad 4 \quad K=\left(\begin{array}{lllllll}12 & 6,1\end{array}\right)$

-D(L21) B2XF6 C2XD3 $1 / 30 \quad 2 / 25 \quad 3 / 501 \quad 5 / 14$

L19 DEG=4 $F=1 \quad A U T=12 \quad P=(1,13,23,2) \quad G I R=4 \quad C N=2,6$

$A=1 \quad 1 \quad 1 \quad 1,34341222 \quad 6,17001640 \quad E=-4 \quad-2 \quad 4-1 \quad 4+124 \quad K=\left(\begin{array}{lllll}13 & 10 & 5 & 1,1\end{array}\right) \quad B 2 X F 5$ $2 / 16.3 / 214$

L20 DEG $=4 \quad F=I A P$ AUT $=4 \quad P=(1,4,24,1) \quad G I R=3 \quad C N=3,4 \quad T=1 \quad$ CUBOCTAHEDRON

$A=1153,3065030460,11023600 \quad E=5-2 \quad 3+0 \quad 3+2 \quad 4 \quad K=(113,2) \quad L(H 7) \quad-D(L 10)$ $4 / 50$

L21 DEG=4 $\quad F=$ IP AUT $=2 \quad P=(1,22,22,12) \quad$ GIR $=3 \quad C N=3,4 \quad$ ANTIPRISM

$A=1 \quad 1 \quad 3 \quad 15,12 \quad 24 \quad 104 \quad 42 \quad 600,14403300 \quad E=4-2 \quad 2-.73205 \quad 3+0 \quad 2+2.732054$

$K=\left(\begin{array}{llll}10 & 1,3\end{array}\right)-D(L 12) \quad 1 / 44 \quad 3 / 205$

L22 $D E G=4 \quad F=I A \quad A U T=2 \quad P=(1,22,222,1) \quad G I R=3 \quad C N=3,4$

$A=1 \quad 1 \quad 1 \quad 11,24 \quad 12 \quad 4 \quad 202 \quad 454,322 \quad 740 \quad E=2-2.73205 \quad 2-2 \quad 3+0 \quad 2+.73205 \quad 2+2 \quad 4$

$\mathrm{K}=(125,1) \quad 1 / 22 \quad 3 / 403$

L23 $D E G=4 \quad F=1 \quad A U T=64 \quad P=(1,4,14,2) \quad G I R=4 \quad C N=2,6 \quad T=1$

$A=1 \quad 1 \quad 1 \quad 1,36303066,17001700 \quad E=-4 \quad 2-2 \quad 6+0 \quad 2+2 \quad 4 \quad K=\left(\begin{array}{llllll}13 & 11 & 5 & 1,\end{array}\right) \quad F 4[B 1]$ $B 2 \star F 7 \quad C 2 \star D 3 \quad 1 / 42 \quad 2 / 110 \quad 3 / 221 \quad 5 / 24$

L24 $D E G=4 \quad F=I \quad A U T=4 \quad P=(1,22,124) \quad G I R=4 \quad C N=3,6$

$A=1 \quad 1 \quad 1 \quad 1,6 \quad 6050 \quad 224 \quad 222,1114 \quad 512 \quad E=2-3 \quad 2-2 \cup 6+1 \quad 4 \quad K=(13 \quad 6) \quad D,(L 13) \quad 1 / 14$ $2 / 43 \quad 3 / 132 \quad 5 / 42$

L25 $\quad D E G=5 \quad F=X T I \quad A U T=86400 \quad P=(1,5,+) \quad G I R=3 \quad C N=6,2 \quad T=2$

$A=137 \quad 17,3700100300700,170037002[F 8]$ SW(F3) SW(F8) D2[C2] F3[B2] $1 / 25$ $2 / 1213 / 6105 / 13$

L26 DEG $=5 \quad F=1 \quad$ AUT $=64 \quad P=(1,14,4,2) \quad G I R=3 \quad C N=4,3$

$A=1 \quad 3 \quad 7 \quad 3,23 \quad 60160 \quad 14 \quad 414,1700 \quad 3700 \quad E=-3 \quad y-1 \quad 2+3 \quad 5 \quad K=(4,62) \quad S W(F 2) \quad S W(F 4)$ $-D(L 18)-D(L 35) F 4[B 2] 1 / 432 / 1143 / 2455 / 61$

L27 DEG $=5$ AUT $=1 \quad P=(1 / 5 / 6) \quad G I R=3 \quad C N=3,4$

$A=111115,1150 \quad 66306 \quad 412,11603106 \quad E=-3 \quad 2-2.73205 \quad 2-1 \quad 2+0 \quad 2+.73205 \quad 2+2 \quad 5$

$\mathrm{K}=(7,1,3) \quad 3 / 225$

L28 DEG $=5 \quad F=I \quad$ AUT $=2 \quad P=(1,122,222) \quad G I R=3 \quad C N=3,4$

$A=1 \quad 1 \quad 5 \quad 1,150 \quad 124 \quad 252 \quad 526,272 \quad 166 \quad E=2-3.73205 \quad 2-1 \quad 2-.26795 \quad 5+1 \quad 5 \quad K=\left(\begin{array}{llll}9 & 4,1\end{array}\right)$ D(L22) $1 / 61 \quad 3 / 413$

L29 $D E G=5 \quad F=1 \quad$ AUT $=2 \quad P=(1,122,222) \quad G I R=3 \quad C N=4,3$

$A=1371,122 \quad 42 \quad 170264,1350724 \quad E=-3 \quad 2-2.73205 \quad 2-1 \quad 2+0 \quad 2+.732052+2 \quad 5$

$\mathrm{K}=\left(\begin{array}{lll}7,3 & 1\end{array}\right) \quad 1 / 13 \quad 3 / 174$ 
TRANSITIVE GRAPHS ON 12 VERTICES (CONTD)

L30 $\quad D E G=5 \quad F=1 \quad$ AUT $=12 \quad P=(1,23,6) \quad G I R=3 \quad C N=4,3$

$A=1 \quad 3 \quad 1 \quad 11,3144 \quad 12 \quad 314 \quad 222,1524 \quad 1342 \quad E=6-2 \quad 3+1 \quad 2+2 \quad 5 \quad K=(6,4 \quad 1) \quad C 2 X D 4 \quad-C 2 \star D 4$ $\begin{array}{llll}1 / 31 & 2 / 27 \quad 3 / 434 & 4 / 17 & 5 / 31\end{array}$

L31 DEG $=5$ AUT $=1 \quad P=(1 / 5 / 6) \quad G I R=3 \quad C N=3,4$

$A=1 \quad 1 \quad 1 \quad 15,1574 \quad 42 \quad 210702,6223406 \quad E=2-2.73205 \quad 2-2 \quad-1 \quad 2+0 \quad 2+.732051135$ $K=\left(\begin{array}{lll}6 & 1,4\end{array}\right) \quad 3 / 503$

L32 DEG=5 $F=I \quad A U T=4 \quad P=(1,122,24) \quad G I R=3 \quad C N=4,3$

$A=1 \quad 1 \quad 1 \quad 3,23 \quad 16 \quad 16 \quad 250144,1630 \quad 1524 \quad E=2-3 \quad 2-2 \quad 4+0 \quad 1 \quad 2+2 \quad 5 \quad K=(7,3 \quad 1) \quad 1 / 15$

$2 / 173 / 3115 / 23$

L33 DEG $=5 \quad F=I \quad A U T=4 \quad P=(1,14,24) \quad G I R=3 \quad C N=4,4$

$A=1 \quad 1 \quad 1 \quad 11,5 \quad 50124 \quad 262 \quad 162,1216 \quad 516 \quad E=3-3 \quad 2-1 \quad 6+1 \quad 5 \quad K=(8 \quad 2,2) \quad-L(F 7) \quad D(L 20)$ $4 / 111$

L34 DEG $=5 \quad F=$ TIA AUT $=120 \quad P=(1,5,5,1) \quad G I R=4 \quad C N=2,6 \quad T=2$

$A=1 \quad 1 \quad 1 \quad 1,174 \quad 72 \quad 66 \quad 56,36 \quad 3700 \quad E=-5 \quad 5-1 \quad 5+1 \quad 5 \quad K=\left(\begin{array}{lllll}10 & 10 & 5 & 1,1\end{array} \quad\right.$ SW(F1) SW(F5) -W6(B2) $-B 2 X F 8$ B2*F8 2/111 $3 / 163$

L35 DEG $=5 \quad F=I A \quad A U T=8 \quad P=(1,14,14,1) \quad G I R=3 \quad C N=3,4$

$A=1 \quad 1 \quad 1 \quad 15,15 \quad 74 \quad 42 \quad 206 \quad 212,1422 \quad 3700 \quad E=2-3 \quad 5-1 \quad 3+1 \quad 3 \quad 5 \quad K=(6 \quad 2,4) \quad S W(F 6)$ SW(F7) -D(L15) B2XF7 2/124 3/416

L36 DEG $=5 \quad F=1 \quad A U T=2 \quad P=(1,122,222) \quad G I R=3 \quad C N=4,4$

$A=1 \quad 1 \quad 1 \quad 5,31 \quad 50 \quad 124216 \quad 116,642 \quad 3122 \quad E=2-3 \quad 2-1.73205 \quad 2-1 \quad 3+1 \quad 2+1.73205 \quad 5$ $K=(7,3) \quad 1 / 7 \quad 3 / 350$

L37 DEG=5 F=TIAP AUT $=10 \quad P=(1,5,5,1) \quad G I R=3 \quad C N=4,4 \quad T=1 \quad$ ICOSAHEDRON $A=1 \quad 3 \quad 5 \quad 3,3150 \quad 114 \quad 22 \quad 560,606 \quad 3700 \quad E=3-2.23607 \quad 5-1 \quad 3+2.23607 \quad 5 \quad K=(5,5)$ SW(E2+) -D(L37) 4/121

TRANSITIVE GRAPHS ON 13 VERTICES

M1 $D E G=0 \quad F=X T V I A P \quad A U T=479001600 \quad P=(1,+) \quad C N=1,13$

M2 DEG $=2 \quad F=T V I P$ AUT $=2 \quad P=(1,2,2,2,2,2,2) \quad G I R=13 \quad C N=3,7 \quad$ POLYGON

$A=1142,201010040400,20020005000$

$E=2-1.94188 \quad 2-1.49702 \quad 2-.709 \angle 1 \quad 2+.24107 \quad 2+1.136132+1.770912 \quad K=\left(\begin{array}{llll}36 & 56 & 35 & 6,\end{array}\right)$ $1 / 1$

M3 $\quad D E G=4 \quad F=V I \quad A U T=4 \quad P=(1,4,44) \quad G I R=4 \quad C N=4,7 \quad T=1$

$A=1 \quad 1 \quad 1 \quad 1,20 \quad 10 \quad 142 \quad 144 \quad 54,1122 \quad 224 \quad 4412 \quad E=4-2.65109 \quad 4+.273894+1.377204$ $K=\left(\begin{array}{lll}18 & 12,) & 1 / 6\end{array}\right.$

M4 DEG=4 F=VI AUT $=2 \quad P=(1,22,22,22) \quad G I R=3 \quad C N=4,5$

$A=1 \quad 1 \quad 3 \quad 15,24 \quad 1244 \quad 102 \quad 400,120035003240$

$E=2-2.20623 \quad 2-1.700812-1.25595 \quad 2-.17097 \quad 2+.42692 \quad 2+2.90704 \quad 4 \quad K=\left(\begin{array}{lll}15 & 4,3\end{array}\right)$ D(M2) $1 / 44$

M5 $D E G=4 \quad F=V I \quad A U T=2 \quad P=(1,22,222,2) \quad G I R=4 \quad C N=3,7$

$A=1 \quad 1 \quad 1 \quad 1,34 \quad 32 \quad 4 \quad 202 \quad 414,222 \quad 2500 \quad 5240$

$E=2-3.43891 \quad 2-.80575 \quad 2-.46814 \quad 2-.360892+1.061702+2.011994 \quad K=\left(\begin{array}{llll}18 & 16 & 5,\end{array}\right)$ $-D$ (M4) $1 / 5$

M6 DEG $=6 \quad F=T V I S \quad A U T=6 \quad P=(1,6,6) \quad G I R=3 \quad C N=5,5 \quad T=1$

$A=13 \quad 1 \quad 15,11 \quad 43 \quad 124 \quad 312 \quad 432,654 \quad 3046 \quad 5360 \quad E=6-2.30278 \quad 6+1.30278 \quad 6 \quad K=(6,6)$ $1 / 15$

M7 $D E G=6 \quad F=V I S \quad A U T=2 \quad P=(1,222,222) \quad G I R=3 \quad C N=5,5$

$A=1 \quad 3 \quad 3 \quad 15,5 \quad 3 \quad 132 \quad 74 \quad 244,1502 \quad 3350 \quad 3560$

$E=2-3.197832-1.96516 \quad 2-1.070102+.070102+.96516 \quad 2+2.19783 \quad 6 \quad K=(6,6) \quad 1 / 64$

M8 $D E G=6 \quad F=V I$ AUT $=2 \quad P=(1,222,222) \quad G I R=3 \quad C N=4,5$

$A=1 \quad 3 \quad 5 \quad 3,1 \quad 1 \quad 174 \quad 172 \quad 164,1152 \quad 2524 \quad 5252$

$E=2-4.14811 \quad 2-.88018 \quad 2-.56468 \quad 2+.51496 \quad 2+.667992+1.410026 \quad K=\left(\begin{array}{lllllll}9 & 4,3\end{array}\right) \quad-M 9$ D(M5) $1 / 16$

M9 $D E G=6 \quad F=V I \quad A U T=2 \quad P=(1,222,222) \quad G I R=3 \quad C N=5,4$

$A=1 \quad 1 \quad 3 \quad 5,3375 \quad 12452 \quad 412,1224 \quad 36047602$

$E=2-2.41002 \quad 2-1.66799 \quad 2-1.51496 \quad 2-.43532 \quad 2-.11982 \quad 2+3.14811 \quad 6 \quad K=\left(\begin{array}{llllll}3,9 & 4\end{array}\right) \quad-M 8$ $-D$ (M5) $1 / 52$ 
TRANSITIVE GRAPHS ON 14 VERTICES

N1 DEG $=0 \quad F=X T V I A P$ AUT $=6227020800 \quad P=(1,+) \quad C N=1,14$

N2 $\quad$ DEG $=1 \quad F=X T I P \quad A U T=46080 \quad P=(1,1,+) \quad C N=2,7 \quad T=1$

$A=1040,2001000400,02000010000$

N3 $D E G=2 \quad F=X T I P \quad A U T=28 \quad P=(1,2,2,2,+) \quad G I R=7 \quad C N=3,8$

$A=1 \quad 142,2050 \quad 02000,0240012003000 \quad$ 2[G2] $1 / 20 \quad 2 / 200$

N4 DEG $=2 \quad F=T I A P$ AUT $=2 \quad P=(1,2,2,2,2,2,2,1) \quad G I R=14 \quad C N=2,7 \quad$ POLYGON

$A=1 \quad 142,201010040 \quad 400,2002000100014000$

$E=-2 \quad 2-1.801942-1.24698 \quad 2-.445042+.445042+1.246982+1.801942$

$K=\left(\begin{array}{lllll}45 & 84 & 70 & 21 & 1,\end{array}\right) \quad B 2 \star G 2 \quad 1 / 2 \quad 2 / 140$

N5 $\quad D E G=3 \quad F=1 \quad A U T=2 \quad P=(1,12,22,22,2) \quad G I R=4 \quad C N=2,7$

$A=1 \quad 1 \quad 1 \quad 12,6 \quad 104200100,24012034005400$

$E=-3 \quad 2-2.24698 \quad 2-.801942-.554962+.55496 \quad 2+.80194 \quad 2+2.246983$

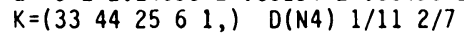

N6 $D E G=3 \quad F=I P \quad A U T=2 \quad P=(1,12,22,22,2) \quad G I R=4 \quad C N=3,7 \quad P R I S M$

$A=1 \quad 1 \quad 1 \quad 12,6 \quad 104200500,240 \quad 120500012400$

$E=2-2.801942-1.445042-.801942+.24698 \quad 2+.55496 \quad 1 \quad 2+2.246983 \quad K=\left(\begin{array}{lllll}33 & 44 & 25 & 6,\end{array}\right)$ B2XG2 $1 / 52 / 201$

N7 DEG $=3 \quad F=T I \quad A U T=24 \quad P=(1,3,6,4) \quad$ GIR $=6 \quad C N=2,7 \quad T=4 \quad$ HEAWOOD GRAPH $A=1 \quad 1 \quad 1 \quad 10,2 \quad 2 \quad 4 \quad 4 \quad 10,12401500460320 \quad E=-3 \quad 6-1.41421 \quad 6+1.414213$ $K=\left(\begin{array}{lllll}33 & 42 & 20 & 6 & 1,1\end{array}\right) \quad 2 / 144$

N8 $\quad D E G=4 \quad F=X I \quad A U T=28 \quad P=(1,22,2,+) \quad G I R=3 \quad C N=4,6$

$A=1 \quad 3 \quad 5 \quad 3,3472 \quad 0200200,400340036007200 \quad 2[G 3] 1 / 104 \quad 2 / 1200$

N9 $D E G=4 \quad F=I A P$ AUT $=2 \quad P=(1,22,22,22,1) \quad G I R=3 \quad C N=4,5 \quad$ ANTIPRISM

$A=1 \quad 1 \quad 3 \quad 15,24 \quad 12 \quad 44 \quad 102 \quad 500,240 \quad 1400620017000$

$E=2-2.24698 \quad 2-1.69202 \quad 2-1.35690 \quad 2-.55496 \quad 0 \quad 2+.80194 \quad 2+3.04892 \quad 4 \quad K=\left(\begin{array}{llll}21 & 10,3\end{array}\right)$ $1 / 602 / 504$

N10 DEG=4 $F=1 \quad$ AUT $=2 \quad P=(1,22,222,12) \quad G I R=4 \quad C N=2,7$

$A=1 \quad 1 \quad 1 \quad 1,32 \quad 34 \quad 1422 \quad 2,4360033003440$

$E=-4 \quad 2-2.24698 \quad 2-.80194 \quad 2-.55496 \quad 2+.55496 \quad 2+.80194 \quad 2+2.246984$

$K=\left(\begin{array}{lllll}24 & 26 & 15 & 6 & 1,\end{array}\right) \quad B 2 \star G 3 \quad 1 / 12 \quad 2 / 164$

N11 $D E G=4 \quad F=1 A \quad A U T=2 \quad P=(1,22,2222,1) \quad G I R=4 \quad C N=3,7$

$A=1 \quad 1 \quad 111,24 \quad 12 \quad 4 \quad 2 \quad 414,1222 \quad 4504320740$

$E=2-3.04892 \quad 2-2.24698 \quad 2-.55496 \quad 0 \quad 2+.80194 \quad 2+1.35690 \quad 2+1.692024 \quad K=\left(\begin{array}{llll}24 & 22 & 5,\end{array}\right)$ $1 / 222 / 214$

N12 DEG=4 $\quad F=I \quad A U T=128 \quad P=(1,4,14,4) \quad G I R=4 \quad C N=3,7 \quad T=1$

$A=1 \quad 1 \quad 1 \quad 1,3630 \quad 6 \quad 6 \quad 30,6003100460013100 \quad E=2-3.60388 \quad 2-.89008 \quad 7+0 \quad 2+2.49396 \quad 4$ $\mathrm{K}=\left(\begin{array}{lllll}24 & 28 & 15 & 3,\end{array}\right)-\mathrm{D}(\mathrm{N} 14) \quad \mathrm{G} 2[\mathrm{~B} 1] \quad 1 / 302 / 1005$

N13 DEG=4 $F=T I \quad A U T=24 \quad P=(1,4,6,3) \quad G I R=4 \quad C N=2,7 \quad T=2$ DUAL OF HEAWOOD

$A=1 \quad 1 \quad 1 \quad 1,3024 \quad 14 \quad 12 \quad 6,22 \quad 2700 \quad 3240 \quad 1540 \quad E=-4 \quad 6-1.41421 \quad 6+1.414214$

$K=\left(\begin{array}{lllll}24 & 24 & 15 & 6 & 1,\end{array}\right) \quad 2 / 154$

N14 $D E G=5 \quad F=I \quad A U T=128 \quad P=(1,14,4,4) \quad G I R=3 \quad C N=5,4$

$A=1 \quad 3 \quad 7 \quad 3,23 \quad 14 \quad 60114 \quad 260,12002500 \quad 720016500$

$E=2-2.603887-12+.109922+3.493965 \quad K=(12,62) \quad-D(N 12) \quad G 2[B 2] \quad 1 / 31 \quad 2 / 207$

N15 DEG $=5 \quad F=1$ AUT $=2 \quad P=(1,122,2222) \quad G I R=3 \quad C N=4,5$

$A=1 \quad 1 \quad 5 \quad 5,11 \quad 5024242 \quad 122,10062412 \quad 3340 \quad 4720$

$E=2-2.69202 \quad 2-2.35690 \quad 2-1.24698 \quad-1 \quad 2+.44504 \quad 2+1.80194 \quad 2+2.048925 \quad K=\left(\begin{array}{lll}15 & 4,3\end{array}\right)$

$D$ (N6) $D$ (N9) $1 / 612 / 1114$

N16 DEG $=5 \quad F=A \quad A U T=1 \quad P=(1 / 5 / 7 / 1) \quad G I R=3 \quad C N=4,5$

$A=1 \quad 1 \quad 111,15606202530,40671020667300$

$E=2-3.21615 \quad 2-1.85926 \quad-1 \quad 2-.38772 \quad 2-.16723 \quad 2+.96917 \quad 2+2.661195 \quad K=\left(\begin{array}{lll}15 & 8,3\end{array}\right)$ $2 / 226$

N17 $D E G=5 \quad F=1 \quad A U T=2 \quad P=(1,122,222,2) \quad G I R=3 \quad C N=4,5$

$A=1 \quad 1 \quad 5 \quad 11,5 \quad 70164 \quad 12 \quad 406,1042 \quad 422720016500$

$E=2-3.24698 \quad 2-1.55496 \quad 2-1.24698 \quad 2-.19806 \quad 2+.44504 \quad 2+1.8019435 \quad K=\left(\begin{array}{llll}15 & 8,3\end{array}\right)$

B2XG3 1/105 2/1201

N18 DEG $=5 \quad F=I \quad$ AUT $=2 \quad P=(1,122,2222) \quad G I R=4 \quad C N=3,7$

$A=1 \quad 1 \quad 1 \quad 1,1667252 \quad 26,11102604 \quad 505012424$

$E=2-4.04892 \quad 2-1.24698-12+.356902+.445042+.69202 \quad 2+1.801945 \quad K=\left(\begin{array}{lll}18 & 16 & 5,\end{array}\right)$

$D(N 11) \quad D(N 5) \quad 1 / 232 / 541$ 


\section{TRANSITIVE GRAPHS ON 14 VERTICES (CONTD)}

N19 $\quad D E G=5 \quad F=1 \quad A U T=2 \quad P=(1,122,222,2) \quad G I R=4 \quad C N=2,7$

$A=1 \quad 1 \quad 1 \quad 1,172 \quad 66 \quad 54 \quad 34,26 \quad 5275007600$

$E=-5 \quad 2-1.80194 \quad 2-1.24698 \quad 2-.445042+.445042+1.24698 \quad 2+1.801945$

$K=\left(\begin{array}{llllll}18 & 20 & 15 & 6 & 1,\end{array}\right) \quad 1 / 13 \quad 2 / 172$

N20 DEG $=6 \quad F=X T I \quad A U T=3628800 \quad P=(1,6,+) \quad G I R=3 \quad C N=7,2 \quad T=2$

$A=13717,37770200600,16003600760017600 \quad$ 2[G4] SW(G4) $1 / 1242 / 1600$

N21 $D E G=6 \quad A U T=1 \quad P=(1 / 6 / 7) \quad G I R=3 \quad C N=5,4$

$A=1 \quad 1 \quad 1 \quad 11,5 \quad 7570 \quad 46 \quad 422,6301456 \quad 3302 \quad 13206$

$E=2-3.21615 \quad-2 \quad 2-1.85926 \quad 2-.38772 \quad 2-.16723 \quad 2+.96917 \quad 2+2.661196 \quad K=\left(\begin{array}{lll}9,6 & 2\end{array}\right)$ $2 / 233$

N22 $D E G=6 \quad F=I \quad A U T=2 \quad P=(1,222,1222) \quad G I R=3 \quad C N=4,5$

$A=1 \quad 3 \quad 1 \quad 1,5 \quad 3 \quad 170 \quad 164 \quad 552,1224 \quad 2612 \quad 4134 \quad 12072$

$E=2-4.04892 \quad 2-1.801942-.44504 \quad 2+.356902+.69202 \quad 2+1.2469826 \quad K=\left(\begin{array}{lll}12 & 6,3\end{array}\right)$ $1 / 1222 / 1206$

N23 DEG=6 $\quad F=1 \quad A U T=2 \quad P=(1,222,1222) \quad G I R=3 \quad C N=4,5$

$A=1 \quad 3 \quad 5 \quad 3,1 \quad 1 \quad 170164 \quad 152,1304264251342472$

$E=2-4.04892-2 \quad 2-1.246982+.356902+.445042+.69202 \quad 2+1.801946 \quad K=\left(\begin{array}{ll}12 & 6,3\end{array}\right)$ $D(N 16) 1 / 46 \quad 2 / 1017$

N24 $D E G=6 \quad F=T I A \quad A U T=720 \quad P=(1,6,6,1) \quad G I R=4 \quad C N=2,7 \quad T=2$

$A=1 \quad 1 \quad 1 \quad 1,1 \quad 1 \quad 174 \quad 172 \quad 166,156136 \quad 76 \quad 17600 \quad E=-6 \quad 6-1 \quad 6+1 \quad 6 \quad K=\left(\begin{array}{llllll}15 & 20 & 15 & 6 & 1,\end{array}\right)$ $\mathrm{SW}(\mathrm{G} 1)-W 7(B 2)-B 2 \times G 4 \quad B 2 \star G 4 \quad 1 / 52 \quad 2 / 173$

N25 DEG $=6 \quad F=1 \quad$ AUT $=2 \quad P=(1,222,1222) \quad G I R=3 \quad C N=4,5$

$A=1 \quad 3 \quad 1 \quad 11,1523 \quad 36214 \quad 222,544 \quad 3142 \quad 545013520$

$E=2-2.69202 \quad 2-2.356902-1.801942-.445042+1.24698 \quad 2 \quad 2+2.04892 \quad 6 \quad K=\left(\begin{array}{lll}9 & 2,6\end{array}\right)$ $1 / 1062 / 1203$

N26 $D E G=6 \quad F=1 \quad A U T=2 \quad P=(1,222,1222) \quad G I R=3 \quad C N=4,5$

$A=1 \quad 1 \quad 3 \quad 5,15 \quad 23 \quad 36214 \quad 222,544 \quad 3142 \quad 5450 \quad 13520$

$E=2-2.69202 \quad 2-2.35690-2 \quad 2-1.24698 \quad 2+.44504 \quad 2+1.801942+2.048926 \quad K=(92,6)$ $1 / 542 / 570$

N27 $\quad D E G=6 \quad F=I A \quad A U T=2 \quad P=(1,222,222,1) \quad G I R=3 \quad C N=4,5$

$A=133 \quad 15,5 \quad 3 \quad 132 \quad 74 \quad 144,1142 \quad 23105460 \quad 17600$

$E=2-3.49396 \quad 6-1 \quad 2-.10992 \quad 2 \quad 2+2.60388 \quad 6 \quad K=\left(\begin{array}{lll}9 & 4,6\end{array}\right) \quad S W(G 3) \quad-D(N 28) \quad 1 / 160 \quad 2 / 1214$

N28 $\quad D E G=6 \quad F=I A \quad A U T=2 \quad P=(1,222,222,1) \quad G I R=3 \quad C N=5,4$

$A=1 \quad 135,3375 \quad 12452 \quad 412,2243204740217600$

$E=2-2.60388-2 \quad 6-1 \quad 2+.10992 \quad 2+3.49396 \quad 6 \quad K=(6,94) \quad S W(G 2)-D(N 17) \quad-D(N 27) \quad 1 / 16$ $2 / 1055$

TRANSITIVE GRAPHS ON 15 VERTICES

01 DEG $=0 \quad F=X \operatorname{XTI} I A P \quad P=(1,+) \quad C N=1,15$

$02 \quad D E G=2 \quad F=X T I P \quad A U T=62208 \quad P=(1,2,+) \quad G I R=3 \quad C N=3,5$

$A=13010,030400240,40002400400024000$ 5[C2] $1 / 20$

$03 \quad D E G=2 \quad F=X T I P \quad A U T=400 \quad P=(1,2,2,+) \quad G I R=5 \quad C N=3,9$

$A=114412,04000500,44020001400010200 \quad 3$ [E2] $1 / 40$

$04 \quad D E G=2 \quad F=T I P$ AUT $=2 \quad P=(1,2,2,2,2,2,2,2) \quad G I R=15 \quad C N=3,8 \quad$ POLYGON

$A=1 \quad 142,201010040 \quad 400,200200010001000024000$

$E=2-1.956302-1.61803 \quad 2-1 \quad 2-.209062+.618032+1.338262+1.827092$

$\mathrm{K}=\left(\begin{array}{lllll}55 & 120 & 126 & 56 & 7,\end{array}\right) \quad 1 / 2$

$05 \quad D E G=4 \quad F=X T I \quad A U T=691200 \quad P=(1,4,+) \quad G I R=3 \quad C N=5,3 \quad T=2$

$A=137 \begin{array}{lllllllllllll} & 3 & 17,0 & 40 & 0 & 140 & 540,1540 & 200 & 4200 & 14200 & 34200 & 3[E 3] & 1 / 44\end{array}$

$06 \quad O E G=4 \quad F=I \quad A U T=4 \quad P=(1,22,24,4) \quad G I R=3 \quad C N=3,5$

$A=1 \quad 1 \quad 1 \quad 11,4 \quad 42 \quad 24 \quad 22 \quad 214,412 \quad 500 \quad 1040 \quad 14240 \quad 16100$

$E=4-2.61803 \quad 4-.38197 \quad 2+.38197 \quad 2+1 \quad 2+2.61803$ 4 $K=\left(\begin{array}{llll}30 & 32 & 10,1\end{array}\right) \quad$ C2XE2 $1 / 60$

$07 \quad D E G=4 \quad F=N T I A \quad A U T=8 \quad P=(1,4,8,2) \quad G I R=3 \quad C N=4,6 \quad T=1$

$A=1 \quad 3 \quad 1 \quad 11,204 \quad 1101442,210 \quad 10603002 \quad 5300 \quad 12440 \quad E=5-2 \quad 4-1 \quad 5+2 \quad 4$

$K=\left(\begin{array}{llll}29 & 24 & 2,2\end{array}\right) \quad L(J 7)$ 
TRANSITIVE GRAPHS ON 15 VERTICES (CONTD)

$08 \quad D E G=4 \quad F=1 \quad$ AUT $=2 \quad P=(1,22,22,22,2) \quad G I R=3 \quad C N=3,5$ $A=1 \quad 1 \quad 3 \quad 15,24 \quad 12 \quad 44 \quad 102400,20015002240700033000$ $E=2-2.16535 \quad 2-2 \quad 4-1 \quad 2-.12920 \quad 2+1.12920 \quad 2+3.16535$ 4 $\quad K=\left(\begin{array}{lllll}28 & 20 & 1,3\end{array}\right) \quad D(04) \quad 1 / 12$

$09 \quad D E G=4 \quad F=I \quad A U T=2 \quad P=(1,22,2222,2) \quad G I R=4 \quad C N=3,8$ $A=1 \quad 1 \quad 1 \quad 1,24 \quad 12442414,1222 \quad 4104220105404340$ $E=2-3.23607 \quad 2-1.827092-1.338262+.20906 \quad 2+1 \quad 2+1.23607 \quad 2+1.956304$ $K=\left(\begin{array}{llll}31 & 36 & 15 & 2,\end{array}\right) \quad 1 / 104$

$010 \quad D E G=4 \quad F=I \quad A U T=2 \quad P=(1,22,222,22) \quad G I R=3 \quad C N=3,5$ $A=1 \quad 1 \quad 1 \quad 11,24 \quad 1254 \quad 122 \quad 2,430403100 \quad 1140026200$ $E=2-2.95630 \quad 2-2 \quad 2-1.20906 \quad 2+.33826 \quad 2+.38197 \quad 2+.827092+2.618034 \quad K=\left(\begin{array}{lllll}30 & 32 & 11,1\end{array}\right)$ $1 / 22$

$011 \quad D E G=4 \quad F=1 \quad A U T=2 \quad P=(1,22,222,22) \quad G I R=4 \quad C N=3,8$ $A=1 \quad 1 \quad 1 \quad 1,34324202 \quad 14,22 \quad 24005200125005240$ $E=2-3.57433 \quad 4-1 \quad 2-.27977 \quad 2+.40898 \quad 2+1 \quad 2+2.44512 \quad 4 \quad K=\left(\begin{array}{lllll}31 & 40 & 25 & 6,1\end{array}\right) \quad 1 / 140$

$012 \quad D E G=4 \quad F=I \quad A U T=4 \quad P=(1,4,224,2) \quad G I R=4 \quad C N=3,8 \quad T=1$

$A=1 \quad 1 \quad 1 \quad 1,24 \quad 1230 \quad 6 \quad 120,5010422104660011600$

$E=2-3.23607 \quad 2-2 \quad 4-.61803 \quad 2+1.23607 \quad 4+1.618034 \quad K=\left(\begin{array}{lllll}31 & 36 & 16 & 2,\end{array}\right) \quad C 2 \star E 2 \quad 1 / 11$

$013 \quad D E G=6 \quad F=I \quad A U T=2 \quad P=(1,222,2222) \quad G I R=3 \quad C N=5,3$

$A=137 \quad 17,1150 \quad 320344,5422510126016504 \quad 15242$

$E=2-2.956302-2.61803 \quad 2-1.209062-.38197 \quad 2+.33826 \quad 2+.827092+36 \quad K=\left(\begin{array}{lllll}13,6 & 4 & 1\end{array}\right)$ IFj 44

$014 \quad D E G=6 \quad F=1 \quad A U T=2 \quad P=(1,222,2222) \quad G I R=3 \quad C N=3,5$

$A=13 \quad 5 \quad 3,1 \quad 41 \quad 134 \quad 72 \quad 104,42346433123260 \quad 23510$

$E=2-3.16535 \quad 2-3 \quad 2-1.12920 \quad 2+.12920 \quad 4+1 \quad 2+2.16535 \quad 6 \quad K=\left(\begin{array}{lllll}15 & 8 & 1,4\end{array}\right) \quad 1 / 121$

$015 \quad D E G=6 \quad F=I \quad A U T=2 \quad P=(1,222,2222) \quad G I R=3 \quad C N=4,5$

$A=1 \quad 3 \quad 1 \quad 1,5 \quad 3 \quad 72 \quad 334 \quad 64,1112 \quad 2224 \quad 1412 \quad 16160 \quad 15150$

$E=2-3.783392-2.61803 \quad 2-.38197 \quad 2+0 \quad 2+.48883 \quad 2+1.54732 \quad 2+1.74724 \quad 6 \quad K=\left(\begin{array}{llll}16 & 8,3\end{array}\right)$ $1 / 16$

$016 \quad D E G=6 \quad F=1 \quad A U T=4 \quad P=(1,24,224) \quad G I R=3 \quad C N=3,5$

$A=1 \quad 3 \quad 5 \quad 13,5 \quad 43 \quad 146 \quad 36 \quad 30,1403300 \quad 3420 \quad 17410 \quad 17240$

$E=2-3 \quad 4-1.61803 \quad 2-1.23607 \quad 4+.61803 \quad 2+3.23607 \quad 6 \quad K=\left(\begin{array}{llll}12 & 4 & 1,7\end{array}\right) \quad 1 / 122$

$017 \quad D E G=6 \quad F=1 \quad A U T=2 \quad P=(1,222,222,2) \quad G I R=3 \quad C N=5,4$

$A=1 \quad 1 \quad 3 \quad 5,33 \quad 75 \quad 124 \quad 52 \quad 204,1402 \quad 2412 \quad 1224 \quad 17200 \quad 37400$

$E=2-2.61803 \quad 2-1.74724 \quad 2-1.54732 \quad 2-.48883 \quad 2-.38197 \quad 2+0 \quad 2+3.78339 \quad 6 \quad K=\left(\begin{array}{llll}10,9 & 4\end{array}\right)$

$-D(010)-D(011) 1 / 52$

$018 \quad D E G=6 \quad F=I \quad A U T=2 \quad P=(1,222,2222) \quad G I R=3 \quad C N=4,5$

$A=1 \quad 1 \quad 3 \quad 5,23 \quad 55 \quad 164 \quad 152 \quad 204,402 \quad 3220 \quad 7410 \quad 13012 \quad 27024$

$E=2-2.827092-2.33826 \quad 2-1.23607 \quad 2-.79094 \quad 2+0 \quad 2+.95630 \quad 2+3.23607 \quad 6 \quad K=\left(\begin{array}{lll}12 & 4,7\end{array}\right)$ $-D(017) 1 / 124$

$019 \quad D E G=6 \quad F=1 \quad A U T=2 \quad P=(1,222,2222) \quad G I R=3 \quad C N=3,5$

$A=1 \quad 1 \quad 1 \quad 11,23 \quad 55 \quad 134 \quad 72 \quad 42,104 \quad 3404 \quad 7202 \quad 132147422$

$E=2-3 \quad 2-2.61803 \quad 2-.827092-.38197 \quad 2-.33826 \quad 2+1.20906 \quad 2+2.95630 \quad 6 \quad K=\left(\begin{array}{llll}13 & 4 & 1,6\end{array}\right)$ D(08) $1 / 13$

020 DEG=6 $F=1 \quad$ AUT $=48 \quad P=(1,24,8) \quad G I R=3 \quad C N=5,3$

$A=1 \quad 3 \quad 1 \quad 11,31 \quad 71 \quad 104 \quad 12 \quad 614,422 \quad 3224 \quad 2442 \quad 15244 \quad 12702 \quad E=8-2 \quad 4+1 \quad 2+3 \quad 6$

$K=(12,74$ i) C2XE $3-C 2 \star E 3 \quad 1 / 64$

$021 \quad D E G=6 \quad F=N T V I \quad A U T=48 \quad P=(1,6,8) \quad G I R=3 \quad C N=4,5 \quad T=1$

$A=1 \quad 3 \quad 1 \quad 1,21 \quad 11 \quad 124 \quad 142 \quad 654,54 \quad 2342 \quad 2524 \quad 15032 \quad 2632 \quad E=5-3 \quad 9+1 \quad 6 \quad K=\left(\begin{array}{llll}16 & 8 & 2,3\end{array}\right)$ $-L(F 8) D(07)$

022 DEG=6 F=I AUT=2 $P=(1,222,2222) \quad G I R=3 \quad C N=3,5$

$A=1 \quad 3 \quad 1 \quad 1,1 \quad 1 \quad 174 \quad 172 \quad 424,1212 \quad 2124 \quad 5052 \quad 121645152$

$E=2-4.57433 \quad 2-1.27977 \quad 2-.59102 \quad 2+0 \quad 4+1 \quad 2+1.44512 \quad 6 \quad K=\left(\begin{array}{lllll}18 & 16 & 5,1\end{array}\right) \quad 1 / 62$

$023 \quad D E G=6 \quad F=I \quad A U T=5184 \quad P=(1,6,26) \quad G I R=4 \quad C N=3,8 \quad T=1$

$A=1 \quad 1 \quad 111,111176176 \quad 160,1016101610161616016160$

$E=2-4.8541010+02+1.854106 \quad K=\left(\begin{array}{lllll}19 & 20 & 10 & 2,\end{array}\right) \quad D(012) \quad D(09) \quad E 2[C 1] 1 / 51$

024 DEG $=6 \quad F=1 \quad A U T=4 \quad P=(1,24,224) \quad G I R=3 \quad C N=4,6$

$A=1 \quad 1 \quad 5 \quad 13,5 \quad 4322 \quad 204 \quad 740,630 \quad 1262 \quad 1514 \quad 646412312$

$E=2-2.61803 \quad 4-2.23607 \quad 2-.38197 \quad 2+0 \quad 4+2.23607 \quad 6 \quad K=\left(\begin{array}{lllll}13 & 4,6\end{array}\right) \quad-D(06) \quad 1 / 15$ 
5. Additional Information. (a) Two graphs are cospectral if their adjacency matrices have the same eigenvalues and multiplicities. We list here all families of cospectral graphs in the catalogue. The complements of each member of a family form another family.

12 vertices: L15 L21 L27 L29.

16 vertices: $\quad$ P33 P49, P35 P45, P61 P88, P63 $\quad$ P72,

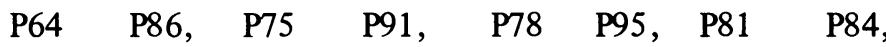

P97 P107, P98 P134, P99 P113 P118, P103 P108,

P105 P141, P111 P112, P120 P136, P124 P137,

P142 P143.

(b) The following graphs are the only ones in the catalogue which are not Cayley graphs:

$$
\text { J7, O7, O21, P20, P52, P93, P110, R38, R147. }
$$

(c) The switching classes of transitive graphs of even order are shown in Table 3. It is easy to show that $X$ and $Y$ are switching equivalent if and only if $\bar{X}$ and $\bar{Y}$ are. Thus each family in Table 3 provides another by complementing each member. However the following graphs are actually switching equivalent to their own complements:

$$
\text { B1, J3, J6, J7, R15, R32, R38, R39, R147, R148, R161, R179. }
$$

Table 3 does not include the following graphs, as they are unique in their switching classes: L10, L16, L37, P74 and P139. It may be worth noticing that each family of cospectral graphs is related also by switching. In fact, two switching equivalent regular graphs of the same degree are necessarily cospectral.

(d) The self-complementary transitive graphs in the catalogue are E2, I4, M6, M7, Q14, Q15, Q18 and Q20.

(e) The connected planar transitive graphs (excluding polygons) with order less than 20 are D4, F6, F7, H7, H10, J6, J11, L10, L13, L20, L21, L37, N6, N9, P10, P16, R10 and R20.

(f) The distance-regular connected graphs in the catalogue, excluding polygons and those with $d>(n-1) / 2$, are H7, I4, J7, J10, L34, L37, M6, N7, N13, N24, 07, O21, P27, P55, P81, P84, P130, Q18, R11 and R173. Of these, only P84 is not distance-transitive.

(g) $\operatorname{Aut}(X)$ will act primitively on $V(X)$ if $n$ is prime or if $X$ has no edges. Excluding complements, the only other examples in the catalogue where this occurs are for $\mathrm{I4}, \mathrm{J} 7, \mathrm{O} 21, \mathrm{P} 55$ and P81.

(h) The following are all those graphs in the catalogue whose arc-transitivity is at least one. We exclude disconnected graphs, polygons, and those whose complements are a disjoint union of complete graphs. 


$$
\begin{aligned}
& \mathrm{H} 7, \mathrm{I} 4, \mathrm{~J} 7, \mathrm{~J} 9, \mathrm{~J} 10, \overline{\mathrm{J}} 7, \mathrm{~L} 20, \mathrm{~L} 23, \mathrm{~L} 34, \mathrm{~L} 37, \overline{\mathrm{L} 30}, \mathrm{M} 3, \mathrm{M} 6, \\
& \text { N7, N12, N13, N24, O7, O12, O21, O23, } \mathrm{O} 20, \overline{\mathrm{O}} 1, \mathrm{P} 12, \mathrm{P} 23, \mathrm{P} 27, \\
& \text { P55, P81, P82, P84, P130, } \overline{\mathrm{P} 55}, \overline{\mathrm{P} 81}, \mathrm{Q} 3, \mathrm{Q} 18, \mathrm{R} 11, \mathrm{R} 28, \mathrm{R} 29, \\
& \text { R88, R90, R171, R173, R126, S14. }
\end{aligned}
$$

(i) The only connected graph in the catalogue which has no Hamiltonian cycle is Petersen's graph (J7), which has Hamiltonian paths and cycles of length 9.

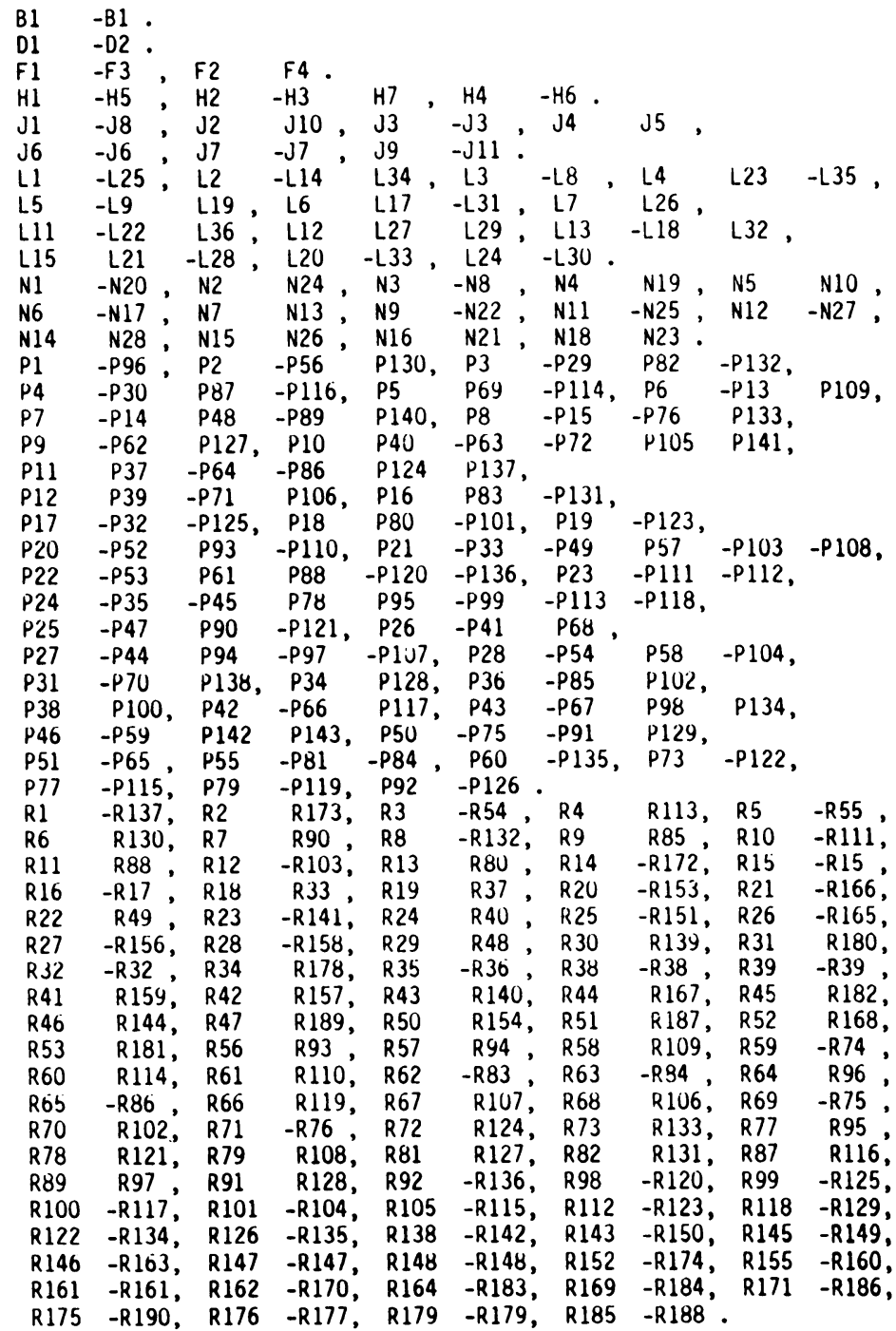

TABLE 3. Switching classes of transitive graphs (-X is the complement of $X)$ 
1. M. BEHZAD \& G. CHARTRAND, Introduction to the Theory of Graphs, Allyn and Bacon, Boston, Mass., 1971.

2. N. BIGGS, Algebraic Graph Theory, Cambridge Tracts in Mathematics No. 67, Cambridge, 1974.

3. C. GODSIL, Neighbourhoods of Transitive Graphs and GRR's, Mathematics Research Report No. 2, University of Melbourne, 1977.

4. J. J. SEIDEL, "Graphs and two-graphs," Proc. 5th Southeastern Conf. on Combinatorics, Graph Theory and Computing, Utilitas Math., Winnipeg, 1974.

5. H. P. YAP, "Point symmetric graphs with $p \leqslant 13$ points," Nanta Math., v. 6, 1973, pp. 8-20. 